\title{
Cosmic-ray Induced Destruction of CO in Star-forming Galaxies
}

\author{
Thomas G. Bisbas ${ }^{1,2}$, Ewine F. van Dishoeck ${ }^{1,3}$, Padelis P. Papadopoulos ${ }^{4,5,6,7}$, László Szúcs ${ }^{1}$, \\ Shmuel Bialy ${ }^{8}$, and Zhi-Yu Zhang ${ }^{7,9}$ \\ ${ }^{1}$ Max-Planck-Institut für Extraterrestrische Physik, Giessenbachstrasse 1, D-85748 Garching, Germany; tbisbas@ufl.edu \\ ${ }^{2}$ Department of Astronomy, University of Florida, Gainesville, FL 32611, USA \\ ${ }^{3}$ Leiden Observatory, Leiden University, P.O. Box 9513, NL-2300 RA Leiden, The Netherlands \\ ${ }^{4}$ School of Physics and Astronomy, Cardiff University, Queen's Buildings, The Parade, Cardiff, CF24 3AA, UK \\ ${ }^{5}$ Research Center for Astronomy, Academy of Athens, Soranou Efesiou 4, GR-115 27 Athens, Greece \\ ${ }^{6}$ Department of Physics, Section of Astrophysics, Astronomy and Mechanics, Aristotle University of Thessaloniki, Thessaloniki 54124, Greece \\ ${ }^{7}$ European Southern Observatory, Headquarters, Karl-Schwarzschild-Strasse 2, D-85748, Garching bei München, Germany \\ 8 Raymond and Beverly Sackler School of Physics \& Astronomy, Tel Aviv University, Ramat Aviv, 69978, Israel \\ ${ }^{9}$ Institute for Astronomy, University of Edinburgh, Royal Observatory, Edinburgh, EH9 3HJ, UK \\ Received 2016 October 26; revised 2017 March 24; accepted 2017 March 24; published 2017 April 19
}

\begin{abstract}
We explore the effects of the expected higher cosmic ray $(\mathrm{CR})$ ionization rates $\zeta_{\mathrm{CR}}$ on the abundances of carbon monoxide (CO), atomic carbon (C), and ionized carbon $\left(\mathrm{C}^{+}\right)$in the $\mathrm{H}_{2}$ clouds of star-forming galaxies. The study of Bisbas et al. is expanded by (a) using realistic inhomogeneous giant molecular cloud (GMC) structures, (b) a detailed chemical analysis behind the $\mathrm{CR}$-induced destruction of $\mathrm{CO}$, and (c) exploring the thermal state of $\mathrm{CR}$ irradiated molecular gas. CRs permeating the interstellar medium with $\zeta_{\mathrm{CR}} \gtrsim 10 \times($ Galactic) are found to significantly reduce the $[\mathrm{CO}] /\left[\mathrm{H}_{2}\right]$ abundance ratios throughout the mass of a $\mathrm{GMC}$. $\mathrm{CO}$ rotational line imaging will then show much clumpier structures than the actual ones. For $\zeta_{\mathrm{CR}} \gtrsim 100 \times$ (Galactic) this bias becomes severe, limiting the usefulness of $\mathrm{CO}$ lines for recovering structural and dynamical characteristics of $\mathrm{H}_{2}$-rich galaxies throughout the universe, including many of the so-called main-sequence galaxies where the bulk of cosmic star formation occurs. Both $\mathrm{C}^{+}$and $\mathrm{C}$ abundances increase with rising $\zeta_{\mathrm{CR}}$, with $\mathrm{C}$ remaining the most abundant of the two throughout $\mathrm{H}_{2}$ clouds, when $\zeta_{\mathrm{CR}} \sim(1-100) \times\left(\right.$ Galactic). $\mathrm{C}^{+}$starts to dominate for $\zeta_{\mathrm{CR}} \gtrsim 10^{3} \times$ (Galactic). The thermal state of the gas in the inner and denser regions of GMCs is invariant with $T_{\text {gas }} \sim 10 \mathrm{~K}$ for $\zeta_{\mathrm{CR}} \sim(1-10) \times$ (Galactic). For $\zeta_{\mathrm{CR}} \sim 10^{3} \times$ (Galactic) this is no longer the case and $T_{\text {gas }} \sim 30-50 \mathrm{~K}$ are reached. Finally, we identify $\mathrm{OH}$ as the key species whose $T_{\text {gas }}$-sensitive abundance could mitigate the destruction of $\mathrm{CO}$ at high temperatures.
\end{abstract}

Key words: astrochemistry - cosmic rays - galaxies: ISM - ISM: abundances - methods: numerical

\section{Introduction}

Molecular hydrogen $\left(\mathrm{H}_{2}\right)$ gas and its mass distribution in galaxies is of fundamental importance in determining their structural and dynamical characteristics, as well as the process of star formation in them. It does not have a permanent dipole moment, and at its lowest energy level $(\sim 510 \mathrm{~K})$, the $S(2-0)$ quadrupole transition in the far-IR wavelength cannot trace the bulk of the $\mathrm{H}_{2}$ molecules, which predominantly lie in the cold $(\lesssim 100 \mathrm{~K})$ phase. The astronomical community has therefore implemented other lines to infer this mass indirectly, typically using $\mathrm{CO}$, the next most abundant molecule after $\mathrm{H}_{2}$ itself with its bright rotational transitions in the millimeter/ submillimeter wavelength regime $\left([\mathrm{CO}] /\left[\mathrm{H}_{2}\right] \sim 10^{-4}\right.$ in the Milky Way, e.g., Lacy et al. (1994), where [] denotes the abundance compared to the H-nuclei number density). Unlike $\mathrm{H}_{2}$, $\mathrm{CO}$ has a permanent dipole moment and rotational transitions with $\Delta J=1$ are allowed, e.g., $\mathrm{CO} J=1-0$ at $115 \mathrm{GHz}$ is the most commonly used as an $\mathrm{H}_{2}$ gas tracer, with higher- $J$ transitions becoming accessible at high redshifts in the age of Atacama Large Millimeter/submillimeter Array (ALMA) at the high altitude of Llano de Chajnantor plateau in Chile. The goal of this work is to explore to what extent $\mathrm{CO}$ remains a good tracer of the molecular gas mass and dynamics in regions with elevated cosmic rays (CRs), such as expected in actively star-forming (SF) galaxies typical for the early universe.
Once the CO $(J=1-0)$ line emission is detected, a scaling factor is used to convert its velocity-integrated brightness temperature (or the line luminosity) to $\mathrm{H}_{2}$ column density on scales of molecular clouds or larger. This method is statistically robust for $M\left(\mathrm{H}_{2}\right) \gtrsim 10^{5} M_{\odot}$ (for an investigation on the physical condition dependencies and the underlying physics of the CO-to- $\mathrm{H}_{2}$ conversion factor, see e.g., Bolatto et al. 2013; Szúcs et al. 2016). This CO-to- $\mathrm{H}_{2}$ method, calibrated in Galactic conditions (Dickman et al. 1986; Solomon et al. 1987), is widely used in extragalactic observations (e.g., Solomon et al. 1997; Chen et al. 2015; Genzel et al. 2015; Gratier et al. 2017). If multi-J CO (or other molecules like $\mathrm{HCN}$ ) line observations reveal average gas densities, temperatures, and/or dynamic states of molecular clouds that differ from those in the Milky Way, there exists a theoretical framework to use appropriately modified $\mathrm{CO}-$ to- $\mathrm{H}_{2}$ conversion factors (e.g., Bryant \& Scoville 1996; Papadopoulos et al. 2012a). All these techniques work as long as CO and other molecules used to study its average conditions (e.g., $\mathrm{HCN}$ ) remain sufficiently abundant in giant molecular clouds (GMCs), typically not much less abundant as in the Galactic GMCs where these techniques have been calibrated. Lowmetallicity $(Z)$ molecular gas, especially when irradiated by strong FUV radiation, was the first $\mathrm{H}_{2}$ gas phase for which early studies showed that the standard techniques actually fail (Pak et al. 1998; Bolatto et al. 1999). This means that low- $Z$ gas in the outer parts of even ordinary spiral galaxies, like the 
Milky Way, may then be in a very CO-poor phase and thus impossible to trace using CO lines (Papadopoulos et al. 2002; Wolfire et al. 2010).

Atomic carbon (C) line emission is another alternative for deducing the molecular gas distribution in galaxies and one that can be as reliable as low- $J$ CO lines. This is because of its widespread emission in $\mathrm{H}_{2}$ clouds, in contrast to what is expected from the classical theory of photodissociation regions (PDRs) (Gerin \& Phillips 2000; Israel \& Baas 2001; Papadopoulos et al. 2004; Bell et al. 2007; Offner et al. 2014; Glover et al. 2015). There are a number of reasons contributing toward $\mathrm{C}$ line emission being fully associated with $\mathrm{CO}$ line emission and having higher emerging flux densities per $\mathrm{H}_{2}$ column density than those of the low-CO rotational lines used as global $\mathrm{H}_{2}$ gas tracers. This led to an early proposal for using the two $\mathrm{C}$ lines, ${ }^{3} P_{1}-{ }^{3} P_{0}\left(W_{\mathrm{CI}, 1-0}\right)$ at $492 \mathrm{GHz}$ and ${ }^{3} P_{2}-{ }^{3} P_{1}\left(W_{\mathrm{CI}, 2-1}\right)$ at $809 \mathrm{GHz}$, and especially the lower frequency line, as routine $\mathrm{H}_{2}$ gas tracers in galaxies for $z \gtrsim 1$ when the lines shift into the millimeter band (Papadopoulos \& Greve 2004; Papadopoulos et al. 2004). Such a method can now be extended in the local universe as imaging at high frequencies can be performed by ALMA (Krips et al. 2016). In our Galaxy, the Vela Molecular Ridge cloud $\mathrm{C}$ shows that atomic carbon can accurately trace the $\mathrm{H}_{2}$ gas mass (Lo et al. 2014). For extragalactic studies, Zhang et al. (2014) find that in the center of the Seyfert galaxy Circinus, the C-traced $\mathrm{H}_{2}$ mass is consistent with that derived from submillimeter dust continuum and multiple- $J$ CO excitation analysis, while $\mathrm{C}$ observations have recently been used to trace the $\mathrm{H}_{2}$ gas mass in distant starbursts at $z \sim 4$ (Bothwell et al. 2017).

The ongoing discussion regarding the widespread $\mathrm{C}$ line emission in molecular clouds, and thus their ability to trace $\mathrm{H}_{2}$ independently of ${ }^{12} \mathrm{CO}$ and ${ }^{13} \mathrm{CO}$ lines, took another turn after the recent discovery that $\mathrm{CR}$ can very effectively destroy $\mathrm{CO}$ throughout $\mathrm{H}_{2}$ clouds, leaving $\mathrm{C}$ (but not much $\mathrm{C}^{+}$) in their wake (Bisbas et al. 2015, hereafter "B15," see also Bialy \& Sternberg 2015). Unlike FUV photons that only do so at the surface of $\mathrm{H}_{2}$ clouds and produce $\mathrm{C}^{+}$rather than $\mathrm{C}$, CRs destroy $\mathrm{CO}$ volumetrically and can render $\mathrm{H}_{2}$ clouds partly or wholly CO-invisible even in interstelar medium (ISM) environments with modestly boosted $\mathrm{CR}$ ionization rates of $\zeta_{\mathrm{CR}} \sim(10-50) \times$ Galactic, where $\zeta_{\mathrm{CR}}$ is the CR ionization rate $\left(s^{-1}\right)$ (Strong et al. 2004a, 2004b). The latter values are expected in typical SF galaxies in the universe (Hopkins \& Beacom 2006; Daddi et al. 2010), currently studied only using CO (e.g., Genzel et al. 2012). For example, Mashian et al. (2013) inferred a CR ionization rate of $\zeta_{\mathrm{CR}} \sim 3 \times 10^{-14} \mathrm{~s}^{-1}$ in their analysis of $\mathrm{CO} / \mathrm{C}^{+}$emissions in the high-redshift HDF 850.1. B15 found that in addition to the ability of $\mathrm{C}$ lines to trace the $\mathrm{CO}$-rich parts of an $\mathrm{H}_{2}$ cloud, they also probe the CO-poor regions. This is of particular interest especially if its lines are to be a viable $\mathrm{H}_{2}$-tracing alternative to $\mathrm{CO}$ lines. In the current work we reexamine these $\mathrm{CR}$-induced effects discussed by $\mathrm{B} 15$ in the much more realistic setting of inhomogeneous $\mathrm{H}_{2}$ clouds, which could affect their "visibility" in $\mathrm{CO}, \mathrm{C}$, and $\mathrm{C}^{+}$ line emission. Furthermore, we discuss in more detail the chemistry behind the $\mathrm{CR}$-control of the $[\mathrm{CO}] /\left[\mathrm{H}_{2}\right]$ abundance ratio and its dependence on the gas temperature, which itself is affected by CRs. The latter proves to be a very important factor that should be taken into account in turbulent-dynamic cloud simulations that explore similar issues.
Models of $\mathrm{CO}$ destruction in cosmic-ray dominated regions (CRDRs) predict that low- $J \mathrm{CO} / \mathrm{C}$ line flux ratios are general low, $<1$. Recent ALMA observations of the Spiderweb galaxy by Gullberg et al. (2016) find that $W_{\mathrm{CO}(7-6)} / W_{\mathrm{CI}, 2-1} \sim 0.2$, which can be potentially explained from the presence of high CR energy densities. Another interesting recent example is the observation of the $W_{\mathrm{CO}(1-0)} / W_{\mathrm{CI}, 1-0} \sim 0.1-0.4$ ratio in the starburst galaxy NGC253 (Krips et al. 2016), which, in association with early $W_{\mathrm{CO}(7-6)}$ observations indicating warm $\mathrm{H}_{2}$ gas (Bradford et al. 2003), could be due to high $\zeta_{\mathrm{CR}}$ values. High CR energy densities are expected to maintain higher gas temperatures even in far-UV-shielded environments. B15 estimate a gas temperature of $\sim 50 \mathrm{~K}$ when the $\mathrm{CR}$ ionization rate, $\zeta_{\mathrm{CR}}$, is boosted to $\sim 10^{3}$ times the mean Galactic value.

In this paper we perform astrochemical simulations of the effects of energy densities higher than those of Galactic CRs on inhomogeneous molecular clouds, using the 3D-PDR code (Bisbas et al. 2012) to infer the distributions of the CO, C, and $\mathrm{C}^{+}$abundances, and of the gas temperature. This is a continuation of the B15 work using much more realistic molecular cloud structures than those of uniform-density or radially varying densities explored previously. Moreover, we now also analyze the chemistry involved in the CR-induced destruction of $\mathrm{CO}$ and its conversion into $\mathrm{C}$ in greater detail. In all of our simulations we assume that the bulk of the $\mathrm{H}_{2}$ gas interacts with CRs throughout the cloud volume (i.e., the $\mathrm{H}_{2}$ gas "sees" CRs, with the same spectrum, throughout the volume of the cloud). While this is not true for some regions deep inside clouds (Rimmer et al. 2012) and can depend on the specifics of magnetic fields (Padovani et al. 2013), it remains a very good approximation for the bulk of $\mathrm{H}_{2}$ clouds in SF galaxies (Papadopoulos et al. 2011).

The paper is organized as follows. In Section 2 we present the setup of our simulations. In Section 3 we present the results of our calculations and in particular how the probability density functions and the abundance distribution of the above key species, as well as the corresponding heating and cooling functions vary under the different conditions explored. In Section 4 we discuss how $\mathrm{OH}$ enhances the $[\mathrm{CO}] /\left[\mathrm{H}_{2}\right]$ abundance ratio at higher temperatures when $\zeta_{\mathrm{CR}}$ increases, and in Section 5 we refer to the impact of our findings in observations. We conclude in Section 6.

\section{Description of Simulations}

We consider a three-dimensional density distribution of a non-uniform giant molecular cloud (GMC) and use the 3D-PDR (Bisbas et al. 2012) code to perform chemistry and full thermal balance calculations and estimate the abundance distribution of chemical species and the gas temperature distribution.

\subsection{Density Distribution}

The inhomogeneous spherical GMC in our models is rendered by a fractal structure with a fractal dimension of $\mathcal{D}=2.4$ constructed using the method described in Walch et al. (2015). It has a radius of $R=10 \mathrm{pc}$ and mass of $M=1.1 \times 10^{5} M_{\odot}$. This corresponds to an average H-nucleus number density of $\langle n\rangle \simeq 760 \mathrm{~cm}^{-3}$, typical for Milky Way GMCs. The central part of the cloud contains a dense region with peak density $\sim 2 \times 10^{4} \mathrm{~cm}^{-3}$. The fractal dimension is in accordance to the clumpiness factor observed in evolved Galactic H II regions (e.g., Sánchez et al. 2010; 
Table 1

Initial Gas-phase Chemical Abundances Used in the Present Paper

\begin{tabular}{lccc}
\hline \hline $\mathrm{H}$ & $4.00 \times 10^{-1}$ & $\mathrm{Mg}^{+}$ & $3.98 \times 10^{-5}$ \\
$\mathrm{H}_{2}$ & $3.00 \times 10^{-1}$ & $\mathrm{C}^{+}$ & $2.69 \times 10^{-4}$ \\
$\mathrm{He}$ & $8.50 \times 10^{-2}$ & $\mathrm{O}$ & $4.90 \times 10^{-4}$ \\
$\mathrm{~S}$ & $1.32 \times 10^{-5}$ & $\cdots$ & $\cdots$ \\
\hline
\end{tabular}

Note. The abundances correspond to solar undepleted abundances (Asplund et al. 2009) and are relative to the total hydrogen nuclei number density.

Walch et al. 2015). In contrast, the fractal dimension is higher for diffuse clouds $(\mathcal{D} \sim 2.8-3.0)$, meaning that they are more uniform (Walch et al. 2015). The chosen dimension of $\mathcal{D}=2.4$ corresponds to a GMC containing non-homogeneously distributed high-density clumps typical of those that eventually undergo star formation. They are therefore expected to be $\mathrm{H}_{2}$-rich, and for the particular Milky Way conditions, also CO-rich. We do not evolve the cloud hydrodynamicaly and in order to resolve its densest parts, we use a smoothed particle hydrodynamics (SPH) setup of the cloud and represent it with $8.33 \times 10^{5}$ particles. $^{10}$

\subsection{D-PDR Initial Conditions}

We use the 3D-PDR code (Bisbas et al. 2012) in order to calculate the abundances of chemical species in the above fractal cloud. 3D-PDR obtains the gas temperature and the abundance distribution of any arbitrary three-dimensional density distribution by balancing various heating and cooling functions (see Section 3.2). For the simulations of this work we use the same chemical network and initial abundances of species as used in the B15 paper. In particular, we use a subset of the UMIST 2012 network (McElroy et al. 2013) consisting of 6 elements $(\mathrm{H}, \mathrm{He}, \mathrm{C}, \mathrm{O}, \mathrm{Mg}, \mathrm{S}), 58$ species, and more than 600 reactions. Table 1 shows the initial abundances used that correspond to undepleted solar abundances with hydrogen mostly in molecular form (Asplund et al. 2009). We chemically evolve the cloud for $t_{\text {chem }}=10^{7}$ years, at which point the system has reached chemical equilibrium. Chemical equilibrium is typically obtained after $t_{\text {chem }} \sim 10^{5}$ years for a cloud in which $\mathrm{H}_{2}$ has already formed, (e.g., Bell et al. 2006), which is comparable to turbulent diffusion timescales for GMCs in ULIRG environments (Xie et al. 1995; Papadopoulos et al. 2004, see also Section 5.1 of B15). For our modeled GMC, we find that the sound crossing time is $\sim 3 \mathrm{Myr}$. On the other hand, the $\mathrm{H}_{2}$ formation time is $t_{\text {chem }}=1 / R n_{\mathrm{H}} \lesssim 5 \mathrm{Myr}$, where $R=3 \times 10^{-18}\left(T_{\text {gas }} / \mathrm{K}\right)^{1 / 2} \mathrm{~cm}^{3} \mathrm{~s}^{-1}$. We therefore do not expect turbulence to strongly affect our results, although hydrodynamical simulations exploring this effect are needed toward this direction (e.g., Glover \& Clark 2016). We include $\mathrm{H}_{2}$ formation on dust grains, but we do not model $\mathrm{CO}$ freeze-out. The effects of different networks and different elemental abundances are presented in Appendix A. The effects show that our trends are robust.

In all simulations we consider an isotropic far-ultraviolet (FUV) radiation field strength of $\chi / \chi_{0}=1$, normalized to the Draine (1978) spectral shape, and the width is equivalent to $2.7 \times 10^{-3} \mathrm{erg} \mathrm{cm}^{-2} \mathrm{~s}^{-1}$ integrated over the $91.2-240 \mathrm{~nm}$ wavelength range (Habing 1968). At the surface of the cloud, the field strength is therefore approximately equal to

\footnotetext{
${ }^{10}$ The density of each particle is calculated using the SPH code SEREN (Hubber et al. 2011).
}

1/4 Draine (Sternberg et al. 2014). We perform a suite of four simulations by varying the $\mathrm{CR}$ ionization rate, $\zeta_{\mathrm{CR}}$ from $10^{-17} \mathrm{~s}^{-1}$ to $10^{-14} \mathrm{~s}^{-1}$, the upper limit of which corresponds to values suggested for the central molecular zone (CMZ) (e.g., Le Petit et al. 2016). For convenience, we normalize $\zeta_{\mathrm{CR}}$ as

$$
\zeta^{\prime} \equiv \zeta_{\mathrm{CR}} / \zeta_{\mathrm{MW}}
$$

where $\zeta_{\mathrm{MW}}=10^{-17} \mathrm{~s}^{-1}$ is the typically adopted ionization rate of the Milky Way. This latter value is $\sim 0.1$ times that observed in the diffuse ISM (e.g., McCall et al. 2003; Dalgarno 2006; Neufeld et al. 2010; Indriolo \& McCall 2012; Indriolo et al. 2015), but close to the heliospheric value $\left(1.45-1.58 \times 10^{-17} \mathrm{~s}^{-1}\right)$ as measured by the Voyager 1 spacecraft (Cummings et al. 2015). Our baseline choice of a value lower than that observed is made under the assumption that CRs in our model do not attenuate as a function of column density; instead, the corresponding ionization rate remains constant everywhere in the cloud. We therefore adopt a baseline value that corresponds to an already attenuated $\zeta_{\mathrm{CR}}$ within denser $\mathrm{H}_{2}$ gas. ${ }^{11}$

\subsection{CR Ionization Rate and $U V$}

High CR ionization rates, on the order of $\zeta^{\prime}=10^{3}$, are expected in starburst environments such as the (ultra-) luminous infrared galaxies (U/LIRGs, i.e., $L_{\mathrm{IR}}>10^{11-10^{12}} L_{\odot}$ Sanders et al. 2003). In these systems the star formation rate (SFR) density, $\rho_{\mathrm{SFR}} \equiv \mathrm{SFR} / V$ (where SFR is in $M_{\odot} \mathrm{yr}^{-1}$ and $V$ is the corresponding volume), is enhanced by a factor up to $\sim 10^{3}$ compared with the Milky Way. This drives a higher CR energy density as $U_{\mathrm{CR}} \propto \rho_{\mathrm{SFR}}$ (Papadopoulos 2010). Enhanced FUV fields are also expected in such environments, although dust attenuation in these metal-rich objects will keep the boost of the average FUV field incident on the $\mathrm{H}_{2}$ clouds lower than proportional to $\rho_{\mathrm{SFR}}$ (Papadopoulos et al. 2014).

In this paper we do not vary the isotropic FUV radiation field in our simulations because we wish to isolate the effects of CRs. We note, however, that chemo-hydrodynamical simulations performed by Glover \& Clark (2016) suggest that if both $\zeta^{\prime}$ and $\chi$ are increased by two orders of magnitude, clouds with mass $M \sim 10^{4} M_{\odot}$ might be dispersed by the thermal pressure that would dominate the gravitational collapse. The attenuation of the FUV radiation is calculated using the method described in Bisbas et al. (2012), which accounts for the attenuation due to dust, $\mathrm{H}_{2}$ self-shielding, $\mathrm{CO}$ self-shielfing, $\mathrm{CO}$ shielding by $\mathrm{H}_{2}$ lines, and $\mathrm{CO}$ shielding by dust.

\section{Results}

\subsection{Dependency of Column Density and Volumetric Mass of Species on $\zeta^{\prime}$}

Our description begins with analyzing the abundance distribution of species in all four different 3D-PDR simulations. Lada \& Blitz (1988), van Dishoeck \& Black (1988), and van Dishoeck (1992) were the first to divide the gas into $\mathrm{CO}$-poor and $\mathrm{CO}$-rich populations based on the abundance ratio of $[\mathrm{CO}] /\left[\mathrm{H}_{2}\right]$. In this work, we adopt the B15 definition,

\footnotetext{
11 A similar approximation has also been made by Narayanan \& Krumholz (2017).
} 
for which $\mathrm{CO}$-deficient refers to gas that fullfills the conditions

$$
\begin{gathered}
{[\mathrm{CO}] /\left[\mathrm{H}_{2}\right]<10^{-5},} \\
{[\mathrm{H} \mathrm{I}] / 2\left[\mathrm{H}_{2}\right]<0.5 .}
\end{gathered}
$$

In this case, the abundance of $\mathrm{CO}$ averaged over the cloud is $\sim 10 \times$ lower than the average value of $\sim 10^{-4}$ typically found in molecular clouds, while the gas remains $\mathrm{H}_{2}$-rich. ${ }^{12} \mathrm{We}$ define the gas to be "CO-rich" when the gas is $\mathrm{H}_{2}$-rich and $[\mathrm{CO}] /\left[\mathrm{H}_{2}\right] \geqslant 10^{-5}$. For comparison with observations, the column density ratios rather than just local abundance ratios are most relevant, since column densities ultimately control the strength of the velocity-integrated line emission.

Figure 1 shows column density plots of $\mathrm{H}_{2}, \mathrm{H} \mathrm{I}, \mathrm{CO}, \mathrm{C}$, and $\mathrm{C}^{+}$species as well as cross-section plots of the gas temperature $\left(T_{\text {gas }}\right)$ at the $z=0 \mathrm{pc}$ plane, all as a function of $\zeta^{\prime}$. We map the SPH particle distribution on a $256^{3}$ grid using the method described in Appendix B. The recovery of $T_{\text {gas }} \sim 10 \mathrm{~K}$ for the $\mathrm{H}_{2}$ gas inside the cloud with $\chi / \chi_{0}=1$ and $\zeta^{\prime}=1$ obtained using our thermal balance calculations is in agreement with the typical temperatures of FUV-shielded $\mathrm{H}_{2}$ observed in our Galaxy (e.g., Polychroni et al. 2012; Nishimura et al. 2015) and found in other simulations (e.g., Glover \& Clark 2012). On the high end of the average CR energy densities and similar gas densities we recover similar $T_{\text {gas }}$ values as in the calculations for CRDRs performed in the past (Papadopoulos et al. 2011, their Figure 1).

We find that as $\zeta^{\prime}$ increases, the column density of molecular hydrogen, $N\left(\mathrm{H}_{2}\right)$ remains remarkably unaffected for $\zeta^{\prime}$ up to $10^{3}$. We note, however, that if we were to evolve the cloud hydrodynamically (e.g., Glover \& Clark 2016), the higher gas temperature of the cloud would act to reduce the number of high-density clumps, thus affecting the underlying total column density distribution and the chemistry itself. $N(\mathrm{H} \mathrm{I})$ remains low and nearly constant with $\zeta^{\prime}$ up to $\zeta^{\prime}=10^{3}$ when $\mathrm{H}_{2}$ starts being significantly destroyed toward H I. These trends further reflect the findings of Bialy \& Sternberg (2015), who use the $\zeta_{\mathrm{CR}} / n_{\mathrm{H}}$ ratio to determine whether the ISM gas is predominantly atomic or molecular. The thin H I shell seen in Figure 1 results from photodissociation by the FUV radiation, and the column density $\sim 10^{21} \mathrm{~cm}^{-2}$ is in agreement with Sternberg et al. (2014) and Bialy \& Sternberg (2016).

The most interesting interplay is between $\mathrm{CO}, \mathrm{C}$, and $\mathrm{C}^{+}$. As can be seen from Figure 1, $N(\mathrm{CO})$ already starts to decrease from $\zeta^{\prime} \simeq 10$. For $\zeta^{\prime} \gtrsim 10^{2}$, it is everywhere approximately one order of magnitude lower than at $\zeta^{\prime}=1$. We note that for $N(\mathrm{CO})$ at $\zeta^{\prime}=10^{2}$ and $10^{3}$ the upper limit of the color bar is already one order of magnitude lower than for $\zeta^{\prime}=1$ and 10 . While $N\left(\mathrm{H}_{2}\right)$ remains high even at $\zeta^{\prime} \simeq 10^{3}$, the large decrease of $N(\mathrm{CO})$ points to a $\mathrm{CO}$-to- $\mathrm{H}_{2}$ conversion factor well above its Galactic value, and one that may well become uncalibratable (see Section 5). At the same time, as CR particles interact with $\mathrm{He}$, they create $\mathrm{He}^{+}$ions that then react with $\mathrm{CO}$ to form $\mathrm{C}^{+}$. The latter further recombines with free electrons to form neutral carbon. On the other hand, $N(\mathrm{C})$ increases already from $\zeta^{\prime} \gtrsim 10$, peaking at $\zeta^{\prime} \sim 10^{2}$. As shown in Figure 2 for the particular comparison between $\zeta^{\prime}=1$ and $10^{2}$, it is remarkable to find that

$$
N\left(\mathrm{H}_{2}\right)_{\zeta^{\prime}=1} \simeq N\left(\mathrm{H}_{2}\right)_{\zeta^{\prime}=100}
$$

\footnotetext{
${ }^{12}$ In B15 the gas fulfilling conditions (2) and (3) was defined as CO-dark.
}

$$
\begin{gathered}
N\left(\mathrm{C}^{+}\right)_{\zeta^{\prime}=1} \simeq N\left(\mathrm{C}^{+}\right)_{\zeta^{\prime}=100}, \\
N(\mathrm{C})_{\zeta^{\prime}=1} \simeq N(\mathrm{C})_{\zeta^{\prime}=100}, \\
N(\mathrm{CO})_{\zeta^{\prime}=1} \simeq 30 N(\mathrm{CO})_{\zeta^{\prime}=100},
\end{gathered}
$$

suggesting that nearly all $\mathrm{CO}$ that has been destroyed by CRs is converted into $\mathrm{C}^{+}$and $\mathrm{C}$. It is evident from Figure 1 that while at $\zeta^{\prime}=1 \mathrm{CO}$ traces the $\mathrm{H}_{2}$ structure very well, it only traces regions of higher column densities at $\zeta^{\prime}=10$, whereas at $\zeta^{\prime}=10^{2}$ it is almost vanished. It is then replaced primarily by $\mathrm{C}$, showing a much better resemblance with the molecular structure.

An insidious aspect of a CR-controlled $[\mathrm{CO}] /\left[\mathrm{H}_{2}\right]$ abundance ratio inside CR-irradiated clouds revealed by Figure 1 is that if one were to perform typical $\mathrm{CO}$ line observations meant to find the $\mathrm{H}_{2}$ mass and also characterize average gas density and temperature via $\mathrm{CO}$ and ${ }^{13} \mathrm{CO}$ line ratios, their analysis would consistently indicate dense and warm gas, located in those cloud regions where $\mathrm{CO}$ manages to survive in a high-CR environment. Yet these routine observations would be totally oblivious to the $\mathrm{CO}$-poor $\mathrm{H}_{2}$ gas mass (and its conditions) that surrounds these CO-rich warm and dense gas "peaks." For $\zeta^{\prime} \geqslant 100$ that would be most of the $\mathrm{H}_{2}$ gas (see Figure 1), an effect that may have wide-ranging implications for galaxies where most of the SF in the universe occurs. Except for dust continuum emission, only $\mathrm{C}$ and $\mathrm{C}^{+}$line imaging could reveal that extra gas mass. Of these two, only $\mathrm{C}$ line imaging offers a practical method using ground-based telescopes, since the very high frequency of the $\mathrm{C}^{+}$line makes it inaccessible for imaging over much of the redshift space where SF galaxies evolve.

Figure 3 shows the total mass of the above species in the simulated GMC as a function of $\zeta^{\prime}$. The total mass of $\mathrm{H}_{2}$ in the GMC remains nearly unchanged for up to $\zeta^{\prime} \sim 10^{2}$. It is expected that for $\zeta^{\prime}>10^{4}$ the GMC will be $\mathrm{H}$ I dominated with only trace amounts of $\mathrm{H}_{2}$ even at the most dense regions. The particular mass of atomic carbon appears to have a local maximum at $\zeta^{\prime} \sim 10^{2}$, at which point the mass of $\mathrm{CO}$ is two orders of magnitude lower than the corresponding value for $\zeta^{\prime}=1$. On the other hand, the mass of $\mathrm{C}^{+}$increases monotonically at all times, while for $\zeta^{\prime}=10^{3}$ we find that $M$ $\left(\mathrm{C}^{+}\right) \simeq M(\mathrm{C})$.

It is interesting to see that the masses of $\mathrm{H} \mathrm{I}$ and $\mathrm{C}^{+}$increase monotonically, with the mass of $\mathrm{C}^{+}$increasing somewhat faster than that of $\mathrm{HI}$. Both of these species are products of CRs interacting with $\mathrm{H}_{2}, \mathrm{CO}$, and $\mathrm{C}$, hence it is expected that by increasing $\zeta^{\prime}$ their abundances will also increase. The observed trend, however, is likely to be a result of additional volumetric (3D) effects.

\subsection{Heating and Cooling Processes}

The 3D-PDR code performs thermal balance iterations and converges when the total heating rate matches the total cooling rate calculated for each position within the cloud. The heating processes considered include the Bakes \& Tielens (1994) grain and $\mathrm{PAH}$ photoeletric heating with the modifications suggested by Wolfire et al. (2003) to account for the revised PAH abundance estimate from the Spitzer data; carbon photoionization heating (Black 1987); $\mathrm{H}_{2}$ formation and photodissociation heating (Tielens \& Hollenbach 1985); collisional de-excitation of vibrationally excited $\mathrm{H}_{2}$ following FUV pumping (Hollenbach \& McKee 1979); heat deposition per CR ionization (Tielens \& Hollenbach 1985); supersonic turbulent decay heating 

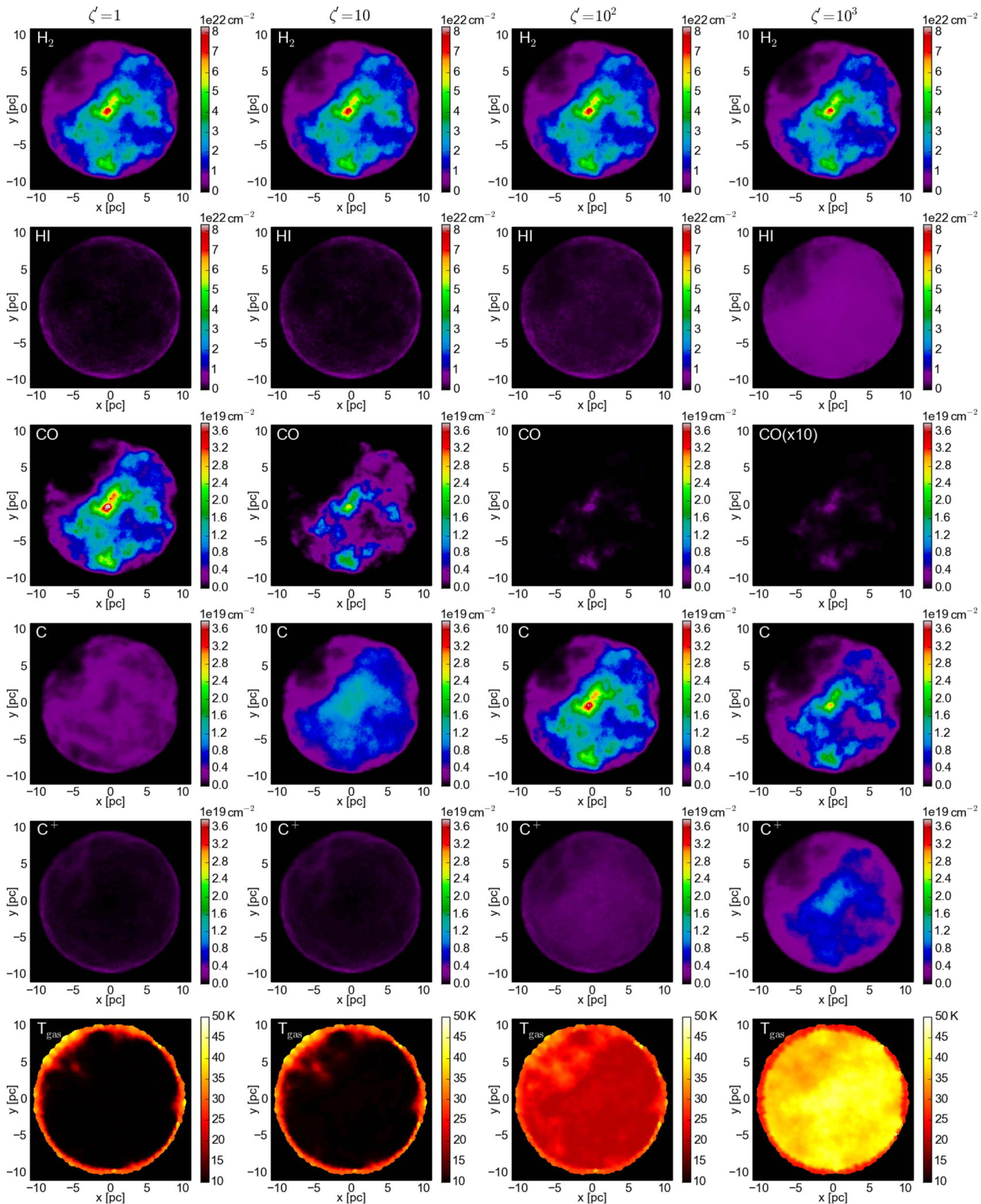

Figure 1. Column density $(N)$ plots of $\mathrm{H}_{2}$ (top row), $\mathrm{H}$ I (second row), $\mathrm{CO}$ (third row), $\mathrm{C}$ (fourth row), and $\mathrm{C}^{+}$(fifth row). The color bar has units of $\mathrm{cm}^{-2}$ and the axes have units of pc. From left to right, $\zeta^{\prime}=1,10,10^{2}, 10^{3}$. Note that $N(\mathrm{CO})$ at $\zeta^{\prime}=10^{3}$ is raised 10 times to make the structure visible. As $\zeta^{\prime}$ increases, $N\left(\mathrm{H}_{2}\right)$ remains remarkably similar, whereas $N(\mathrm{CO})$ is reduced by approximately one order of magnitude. At the same time, $N(\mathrm{C})$ peaks for $\zeta^{\prime}=10^{2}$, while for $\zeta^{\prime}>10^{2}$ it is transformed into $\mathrm{C}^{+}$. It is interesting to note that $N(\mathrm{C})$ at $\zeta^{\prime}=10^{2}$ is approximately equivalent to $N(\mathrm{CO})$ at $\zeta^{\prime}=1$. The bottom row shows cross sections of the gas temperature at the $z=0 \mathrm{pc}$ plane. The color bar there has units of K. The gas temperature at the interior of the cloud increases with $\zeta^{\prime}$, reaching values of up to $\simeq 50 \mathrm{~K}$. For $\zeta^{\prime}=1, T_{\text {gas }} \simeq 10 \mathrm{~K}$ in the cloud center as observed in Milky Way. In all cases the external shell is irradiated by the isotropic FUV radiation, and thus its temperature is determined by that interaction. 

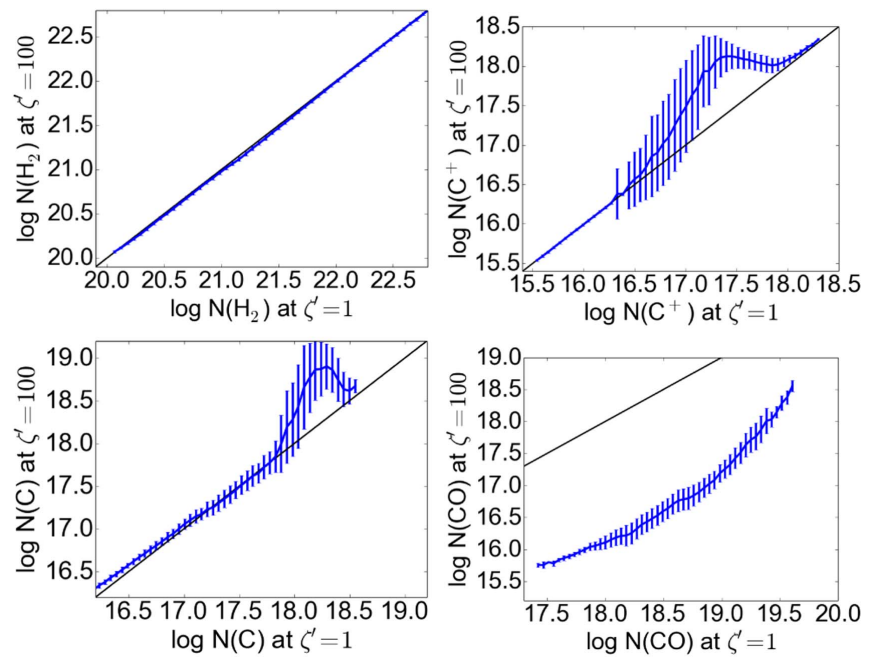

Figure 2. Comparison of column densities at $\zeta^{\prime}=1$ vs. $\zeta^{\prime}=10^{2}$ for $\mathrm{H}_{2}$ (top left), $\mathrm{C}^{+}$(top right), $\mathrm{C}$ (bottom left), and $\mathrm{CO}$ (bottom right). These four panels illustrate Equations (4) and (5), extracted from the simulations shown in Figure 1. The black solid line is the $y=x$ relation to guide the eye. The error bars correspond to $1 \sigma$ standard deviation. These plots show that nearly all $\mathrm{CO}$ that has been destroyed by $\mathrm{CRs}$ is converted into $\mathrm{C}$ and $\mathrm{C}^{+}$, while $\mathrm{H}_{2}$ remains remarkably unaffected.

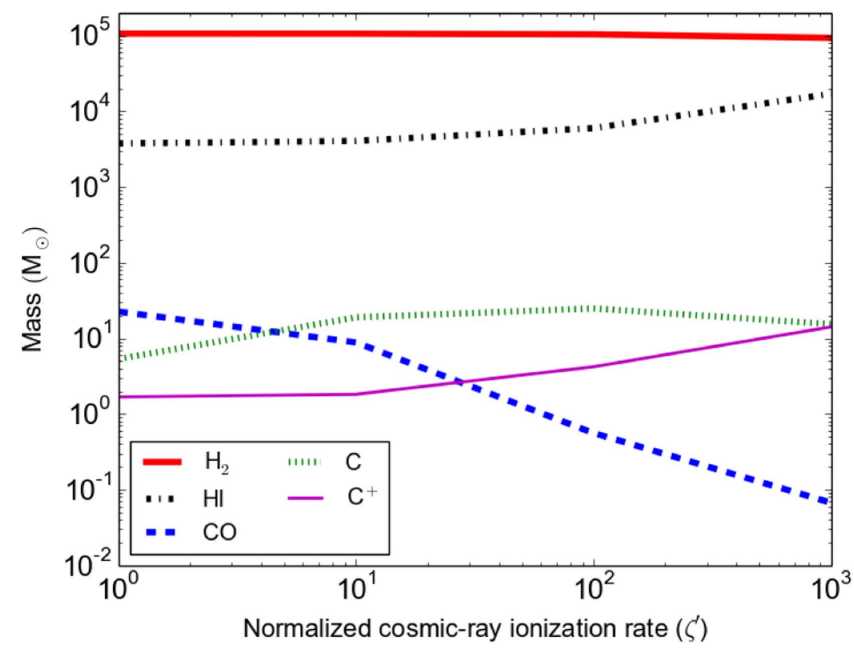

Figure 3. Dependency of $M\left(\mathrm{H}_{2}\right)$ (red thick solid line), $M(\mathrm{H} \mathrm{I})$ (black dottdashed line), $\mathrm{M}(\mathrm{CO})$ (blue dashed line), $\mathrm{M}(\mathrm{C})$ (green dotted line), and $\mathrm{M}\left(\mathrm{C}^{+}\right)$ (magenta thin solid line) as a function of $\zeta^{\prime}$. As $\zeta^{\prime}$ increases, $M\left(\mathrm{H}_{2}\right)$ monotonically decreases, while $M(\mathrm{H} \mathrm{I})$ and $\mathrm{M}\left(\mathrm{C}^{+}\right)$monotonically increase. $\mathrm{M}(\mathrm{C})$ appears to have a local maximum at $\zeta^{\prime} \sim 10^{2}$. For $\zeta^{\prime} \gtrsim 10^{2}, \mathrm{M}(\mathrm{CO})$ is $\sim 2$ orders of magnitude lower than that of $\zeta^{\prime}=1$.

(Black 1987); exothermic chemical reaction heating (Clavel et al. 1978); and gas-grain collisional coupling (Burke \& Hollenbach 1983). The particular $\mathrm{H}_{2}$ formation rate is calculated using the treatment of Cazaux \& Tielens (2002) and Cazaux \& Spaans (2004). The turbulent heating that is included in 3D-PDR is $\propto v_{\text {turb }}^{3} / L$, where $v_{\text {turb }}=1.5 \mathrm{~km} \mathrm{~s}^{-1}$ and $L=5 \mathrm{pc}$. These values are constant throughout all calculations, giving $v_{\text {turb }}^{3} / L \sim 2 \times 10^{-4} \mathrm{~cm}^{2} \mathrm{~s}^{-3}$. Our chosen $v_{\text {turb }}$ is equal to what is expected from the Larson relation and its subsequent observational study by Solomon et al. (1987). This turbulent heating term assumes that turbulence is driven at the largest scale of the cloud (Heyer \& Brunt 2004; Padoan et al. 2009).
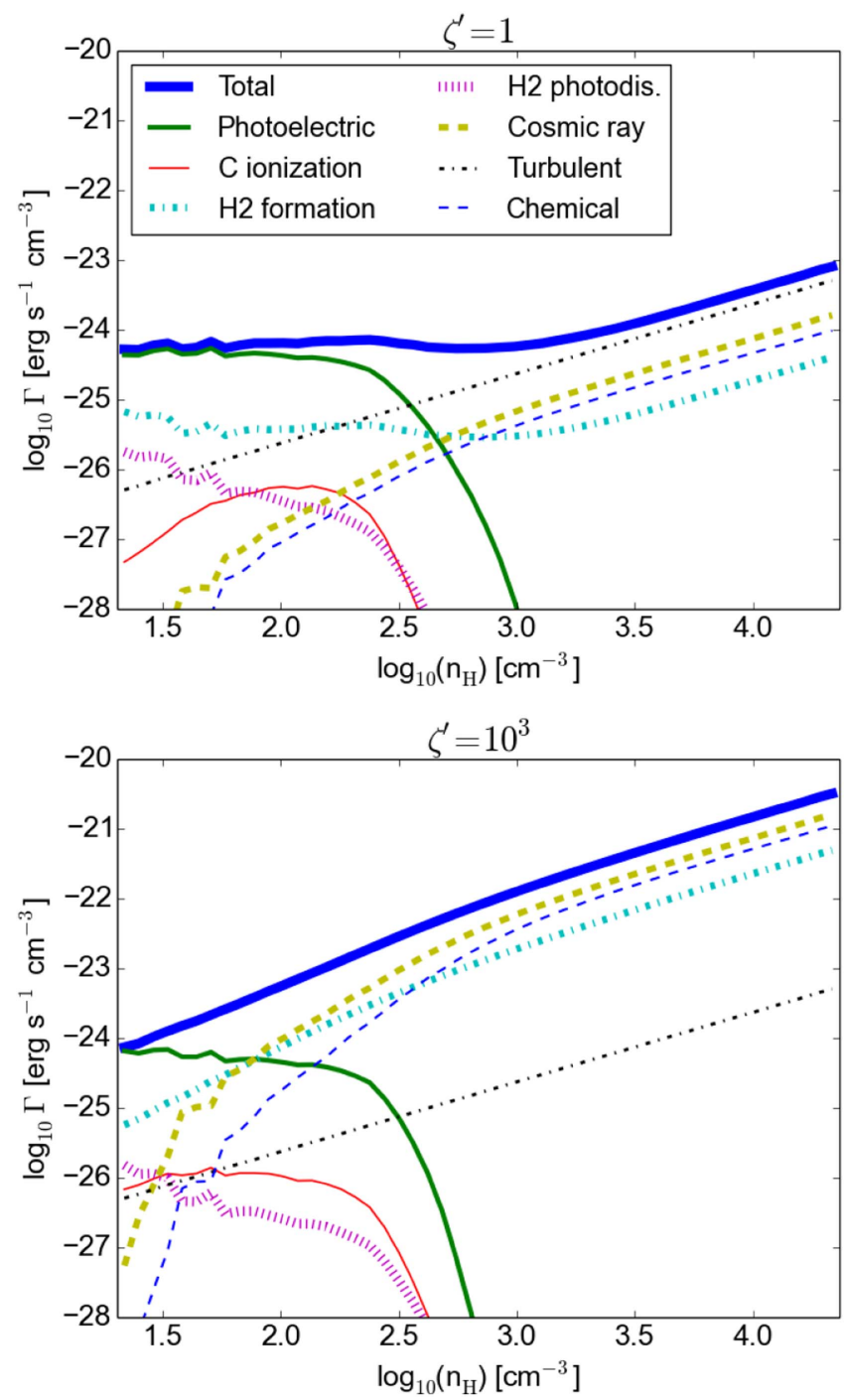

Figure 4. Heating processes for $\zeta^{\prime}=1,10^{3}$. The curves correspond to the mean values for the entire density distribution. For low densities that are located at low visual extinction, heating is in both cases predominantly a result of the photoelectric effect that is due to the interaction of the isotropic FUV field and dust grains. For higher densities, at $\zeta^{\prime}=1$ heating due to turbulent dissipation is the main contributor to the total heating rate, while at $\zeta^{\prime}=10^{3}$ cosmic ray, chemical, and $\mathrm{H}_{2}$ formation heating are responsible for the increase of $T_{\text {gas }}$.

The gas primarily cools as a result of the collisional excitation, subsequent $\mathrm{C}^{+}, \mathrm{C}$, and $\mathrm{O}$ fine-structure line emission, and emission that is due to rotational transitions of CO. The cooling rate of each processes are estimated using a 3D escape probability routine. The details are described in Bisbas et al. (2012) and the data-files are adopted from the Leiden Atomic and Molecular Database (LAMDA; Schöier et al. 2005). ${ }^{13}$ We use a macroturbulent expression to account for the optical depth (Papadopoulos \& Seaquist 1999, and Appendix A of B15).

For densities $n_{\mathrm{H}} \lesssim 10^{2} \mathrm{~cm}^{-3}$ located mainly at the outer regions of the $\mathrm{GMC}$, heating comes predominantly from photoelectrons that are produced as a consequence of the isotropic FUV radiation field (see Figure 4). For $10^{2} \lesssim n_{\mathrm{H}} \lesssim 10^{3} \mathrm{~cm}^{-3}$ and for all $\zeta^{\prime}$, we find that heating

\footnotetext{
$\overline{13}$ http://home.strw.leidenuniv.nl/ moldata/
} 

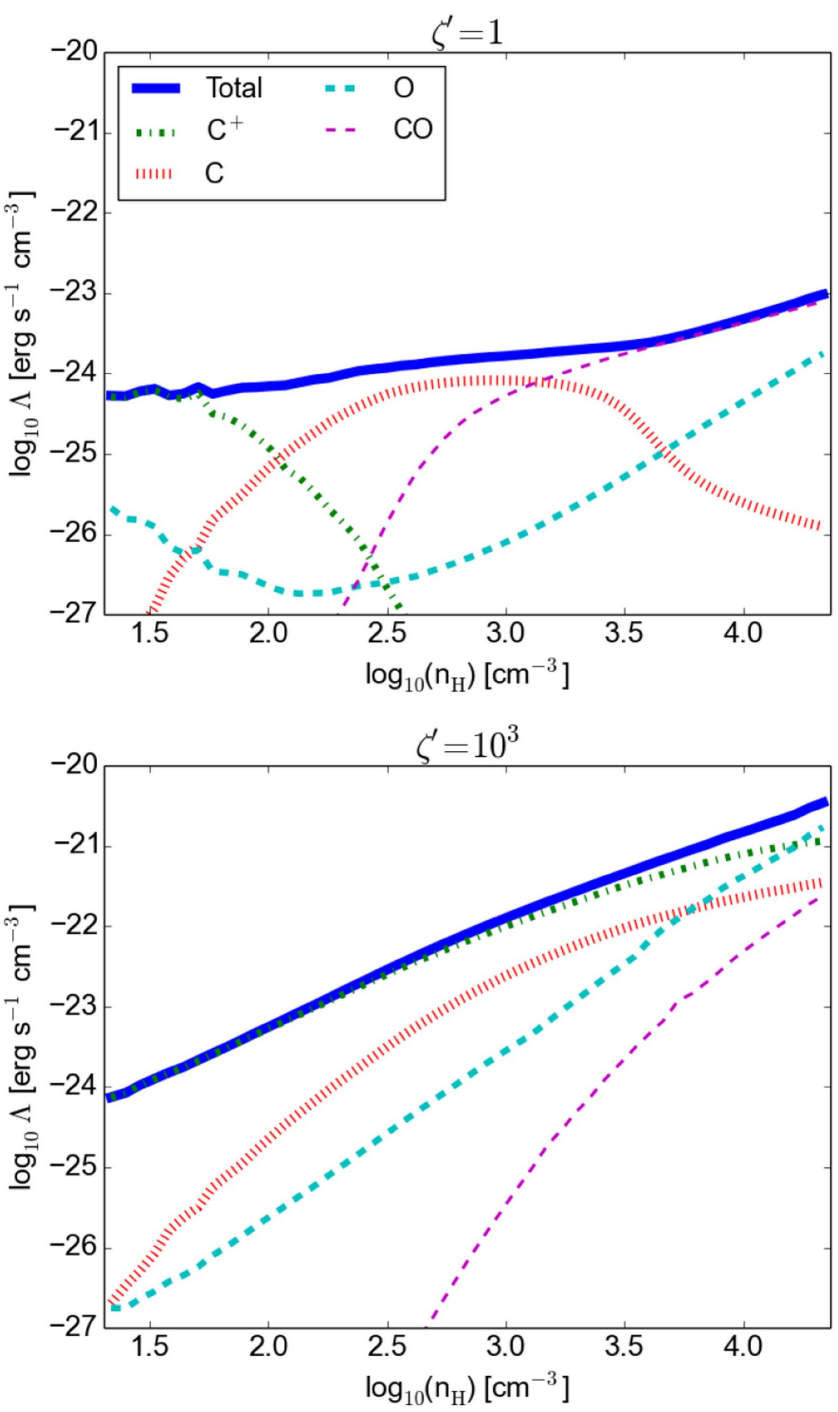

Figure 5. Cooling processes for $\zeta^{\prime}=1,10^{3}$. The curves correspond to the mean values for the entire density distribution. At $\zeta^{\prime}=1$, low-density gas is cooling as a result of the emission of the $\mathrm{C}^{+} 158 \mu \mathrm{m}$ fine-structure line, intermediate densities are due to $\mathrm{C}$, whereas gas at higher densities is due to $\mathrm{CO}$ rotational lines. However, for $\zeta^{\prime}=10^{3}$, the reaction with $\mathrm{He}^{+}$has so severely destroyed $\mathrm{CO}$ and partially $\mathrm{C}$ that over the entire density distribution the main coolants are $\mathrm{C}^{+}$up to $\sim 3 \times 10^{3} \mathrm{~cm}^{-3}$ and $\mathrm{O}$ for higher densities.

results predominantly from contributions by photoelectrons, dissipation of turbulence, exothermic reactions that are due to recombinations of $\mathrm{HCO}^{+}, \mathrm{H}_{3}^{+}, \mathrm{H}_{3} \mathrm{O}^{+}$and ion-neutral reactions of $\mathrm{He}^{+}+\mathrm{H}_{2}$ (chemical heating), energy deposition due to $\mathrm{CR}$ reactions and heating due to $\mathrm{H}_{2}$ formation. For higher densities and for $\zeta^{\prime}=1$, heating results from the turbulence with smaller contributions from CRs and chemical heating. As $\zeta^{\prime}$ increases, however, we find that CRs dominate all other heating mechanisms. The chemical heating also contributes significantly. The latter results from the abundance increase of all participating ions, which is due to reactions ignited by the high CR energy density. This is reflected in the lower panel of Figure 4, where we show the heating functions at $\zeta^{\prime}=10^{3}$.

Likewise, cooling depends on $n_{\mathrm{H}}$ and $\zeta^{\prime}$ (see Figure 5). In particular, for all $\zeta^{\prime}$ we find that at low densities, cooling results predominantly from $\mathrm{C}^{+}$, which-along with photoelectric heating - controls the gas temperature at the outer shell of the GMC. The increase in CR ionization rate results in the increase in $\mathrm{C}^{+}$abundance and hence its cooling efficiency, which in turn decreases the gas temperature. This is the reason why the gas temperature is lower at low $A_{V \text {,eff }}$ (see Section 3.3) with increasing CRs (see Section 3.4). This result has been further reproduced by $1 \mathrm{D}$ calculations confirming the importance of the $\left[\mathrm{C}^{+}\right]$increase. For $\zeta^{\prime} \sim 10^{3}$, we find that $\mathrm{C}^{+}$cooling dominates for densities of up to $n_{\mathrm{H}} \sim 10^{3} \mathrm{~cm}^{-3}$. On the other hand, cooling due to $\mathrm{C}$ is important for $\zeta^{\prime} \lesssim 10^{2}$, particularly for densities between $10^{2} \lesssim n_{\mathrm{H}} \lesssim 10^{3.5}$. Finally, for $n_{\mathrm{H}} \gtrsim 10^{3.5} \mathrm{~cm}^{-3} \mathrm{CO}$ rotational lines contribute predominantly in the gas cooling for $\zeta^{\prime} \lesssim 10^{2}$, with $\mathrm{O}$ to become substantially important for high densities $\left(n_{\mathrm{H}}>10^{3.5}\right)$ and high $\mathrm{CR}$ ionization rates $\left(\zeta^{\prime} \sim 10^{3}\right)$, although it is not a main coolant in all other cases.

Dust temperatures are calculated for each SPH particle using the treatment of Hollenbach et al. (1991) for the heating that is due to the incident FUV photons. This approach is further modified to include the attenuation of the IR radiation as described by Rowan-Robinson (1980). Since the UV radiation on the surface of the cloud is approximately 1/4 Draine (see Section 2.2), the maximum dust temperature we find is $T_{\text {dust }} \sim 12 \mathrm{~K}$, located at large radii. We impose a floor dust temperature of $10 \mathrm{~K}$, which is consistent with the average lowest temperatures observed (e.g., Planck Collaboration et al. 2016). We can therefore assume that the dust temperature in the entire cloud is approximately uniform and equal to $T_{\text {dust }}=10 \mathrm{~K}$.

In regions with densities exceeding $10^{4} \mathrm{~cm}^{-3}, \mathrm{CO}$ freeze-out onto dust grains may become an important process, and the $\mathrm{CO}$ abundance in the gas phase can be sufficiently reduced, which affects its emissivity. CR-induced (photo-)desorption can then bring a small fraction of this gas back to gas phase. Our results would not be altered if we were to include this process. This is because only $\sim 0.4 \%$ of the total mass of the simulated cloud has densities exceeding $10^{4} \mathrm{~cm}^{-3}$ and the corresponding $\mathrm{CO}$ abundance never exceeds $\sim 16 \%$ of the total $\mathrm{CO}$ abundance throughout the cloud (for $\zeta^{\prime}=10^{2}$, whereas for all other cases, it is well below $\sim 10 \%$ ). Moreover, in GMCs, typically only small $\mathrm{H}_{2}$ gas mass fractions reside at regions with $n_{\mathrm{H}}>10^{4} \mathrm{~cm}^{-3}$, making $\mathrm{CO}$ freeze of little importance for the bulk of their mass.

\subsection{Probability Density Functions}

Figure 6 shows mass-weighted probability density distribution functions (PDFs) for each simulation. In these plots it can be seen how the effect of $\mathrm{CO}$ destruction operates volumetrically, particularly when applying conditions (2) and (3). In all plots, the non-shaded part corresponds to CO-rich densities, the light shaded part to all $\mathrm{H}_{2}$-rich but $\mathrm{CO}$-deficient densities, and the dark shaded part to all $\mathrm{HI}$-rich densities. It is interesting to compare the $\mathrm{CO}$-rich, $\mathrm{CO}$-deficient, and $\mathrm{HI}$ regimes with those predicted by B15 from one-dimensional calculations. For this purpose, we also plot in each case the limits for the CO-deficient (vertical solid) and $\mathrm{H}$ I-rich (vertical dashed) regions as indicated in the B15 parameter plot (their Figure 1). For $\zeta^{\prime}=1, \mathrm{~B} 15$ find that for densities $n_{\mathrm{H}} \lesssim 25 \mathrm{~cm}^{-3}$ the gas will be CO-deficient. However, in our 3D simulations we find that for densities up to this value, the gas will also be in $\mathrm{H}$ I form. The $\mathrm{CO}$-deficient $/ \mathrm{H}_{2}$-rich density range now lies in $25 \lesssim n_{\mathrm{H}} \lesssim 200 \mathrm{~cm}^{-3}$ (Figure 6(a)). This difference occurs because of the additional effect of photodissociation of $\mathrm{CO}$, which is due to the isotropic FUV radiation. This 

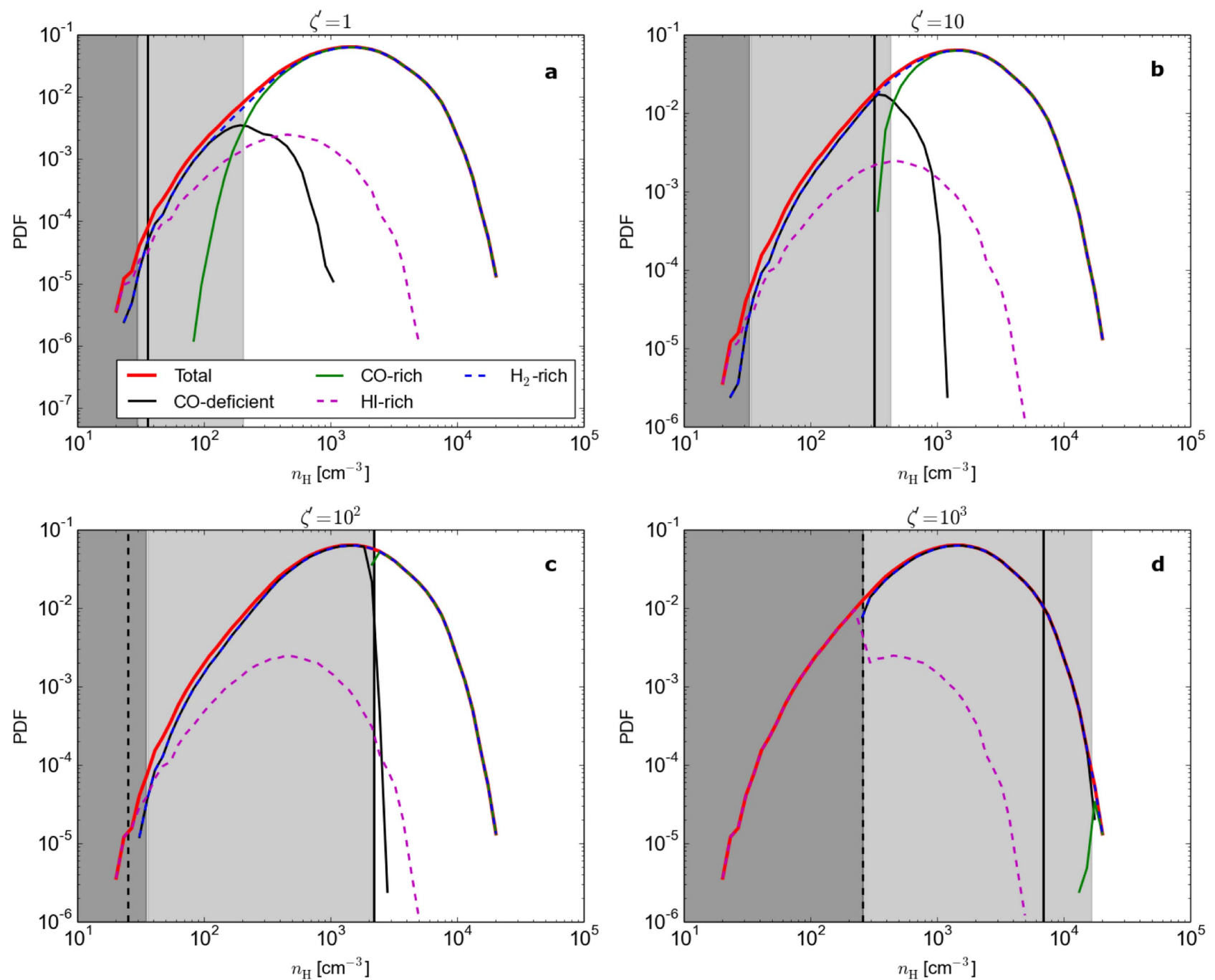

Figure 6. Mass-weighted probability density distribution functions (PDF) of the GMC at different $\zeta^{\prime}$. In panel (a) $\zeta^{\prime}=1$, in panel (b) $\zeta^{\prime}=10$, in panel (c) $\zeta^{\prime}=10^{2}$, and in panel (d) $\zeta^{\prime}=10^{3}$. The red solid line corresponds to the PDF of the total H-nucleus number density of the SPH particles comprising the cloud. The black line corresponds to the PDF of the CO-deficient gas where $[\mathrm{CO}] /\left[\mathrm{H}_{2}\right]<10^{-5}$, and the green line corresponds to PDF of the CO-rich gas. The density range of the COdeficient gas is light shaded. The PDF blue line corresponds to the $\mathrm{H}_{2}$-rich gas where $[\mathrm{H} \mathrm{I}] / 2\left[\mathrm{H}_{2}\right]<0.5$, whereas magenta is for the $\mathrm{H}$ I-rich gas. The density range of the latter is dark shaded. For comparison, vertical lines mark the $[\mathrm{CO}] /\left[\mathrm{H}_{2}\right]=10^{-5}$ (vertical solid) and $\mathrm{H} \mathrm{I} / 2 \mathrm{H}_{2}=0.5$ (vertical dashed) densities as calculated by B15. Owing to the additional photodissociation of CO by the $\chi / \chi_{0}=1 \mathrm{FUV}$ isotropic field (normalized according to Draine 1978), we find a slightly different density range for the CO-deficient gas at $\zeta^{\prime}=1$, as such low densities are found at low visual extinction (see Figure 7). For $\zeta^{\prime}>10$ we obtain very good agreement with the prediction of $\mathrm{B} 15$.

radiation is more effective at lower densities that are located at the outer parts of the cloud (larger radii). This radiation also creates some additional amount of $\mathrm{HI}$ at the outer shell of the cloud, in addition to the CR interaction, as a result of the photodissociation of $\mathrm{H}_{2}$.

The fact that lower densities are located mostly at the outer parts of the cloud is verified in Figure 7, where we correlate the effective visual extinction (e.g., Glover et al. 2010; Offner et al. 2013), $A_{V \text {,eff }}$, defined as

$$
A_{V, \text { eff }}=-0.4 \ln \left(\frac{1}{\mathcal{N}_{\ell}} \sum_{i=1}^{\mathcal{N}_{\ell}} e^{-2.5 A_{V}[i]}\right),
$$

with the $n_{\mathrm{H}}$ number density. This $A_{V \text {,eff }}$ is different from the observed visual extinction. When looking toward the center of a spherically symmetric cloud, this expression would give half of the observed $A_{V}$, which is calculated from one edge of the cloud to the other. In the above equation, $\mathcal{N}_{\ell}$ corresponds to the number of HEALPix (Górski et al. 2005) rays we used and which is equal ${ }^{14}$ to 12 . Indeed, from Figure 7 we find that densities of $n_{\mathrm{H}} \lesssim 200 \mathrm{~cm}^{-3}$ have a mean visual extinction of $A_{V \text {,eff }} \lesssim 0.8 \mathrm{mag}$ and are located mainly at the outer shell of the cloud (see also Wu et al. 2015). They are therefore affected by the FUV radiation.

For $\zeta^{\prime}=10$ and $10^{2}$ (Figures 6(b) and (c)) we find very good agreement with the B15 parameter plot in estimating the density range of the CO-deficient gas. As discussed above, this is the range of $\mathrm{CR}$ ionization rates for which we obtain high abundances of $\mathrm{C}$ while the gas remains almost entirely $\mathrm{H}_{2}$-rich. As can be seen in both cases, the $[\mathrm{CO}] /\left[\mathrm{H}_{2}\right]$ ratio is $\gtrsim 10^{-5}$ only for moderate/high densities (i.e., $n_{\mathrm{H}} \gtrsim 500 \mathrm{~cm}^{-3}$ and $n_{\mathrm{H}} \gtrsim 2 \times 10^{3} \mathrm{~cm}^{-3}$, respectively, see

\footnotetext{
14 Following the analysis by Offner et al. (2013), we do not expect our result to sensitively depend on the chosen angular resolution. See also Clark et al. (2012).
} 


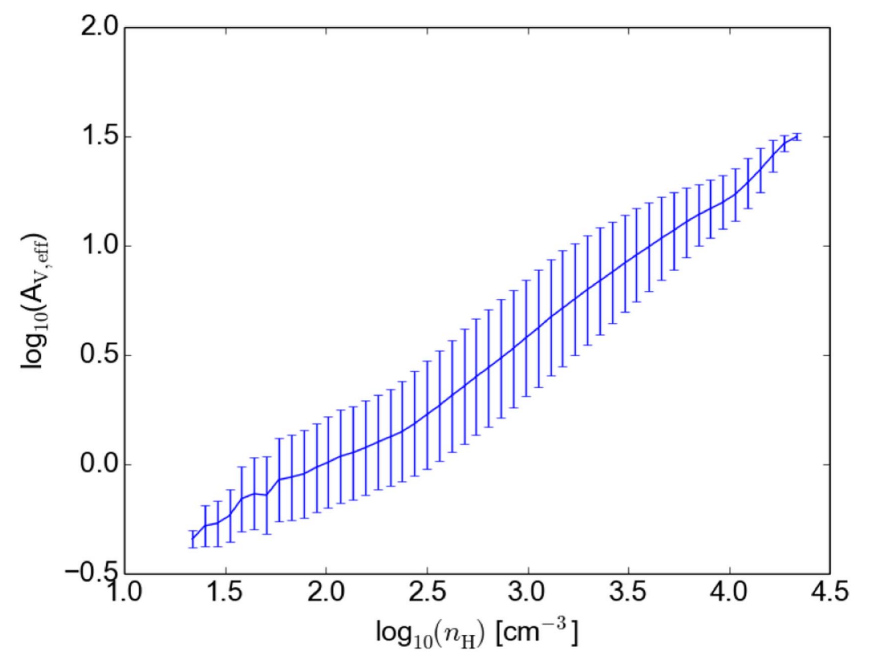

Figure 7. Correlation of $n_{\mathrm{H}}$ with $A_{V \text {, eff }}$ for the present GMC. The error bars correspond to $1 \sigma$ standard deviation. Low densities have a low effective visual extinction as they are primarily located at larger radii (cloud edge), whereas high densities are well shielded from the isotropic FUV radiation field.

Bialy \& Sternberg (2015) for an analytical description concerning the dependency of the $[\mathrm{CO}] /\left[\mathrm{H}_{2}\right]$ ratio as a function of $\zeta_{\mathrm{CR}} / n_{\mathrm{H}}$ ).

As seen in Figure 6(d), for $\zeta^{\prime}=10^{3}$ we find that the density range dominated by $\mathrm{H} \mathrm{I}$ is in agreement with the $\mathrm{B} 15$ parameter plot at a remarkable precision. However, the density range of the CO-deficient gas is now wider. Although for this $\zeta^{\prime}, \mathrm{B} 15$ predict that the CO-deficient gas will be observed in the $300 \lesssim n_{\mathrm{H}} \lesssim 7 \times 10^{3} \mathrm{~cm}^{-3}$ density range, the corresponding upper limit that we find here is $\sim 1.5 \times 10^{4} \mathrm{~cm}^{-3}$. As discussed in $\mathrm{B} 15$, the turnover point is sensitive to the gas temperature obtained from the thermal balance, whereas the latter is also sensitive to the cooling functions, which depend on the density distribution. We therefore assign this discrepancy to the additional 3D effects that cannot be modeled with corresponding 1D calculations.

\subsection{Abundances Distribution and Gas Temperatures}

In Figure 8 we show the gas temperature, $T_{\text {gas }}$, versus the $n_{\mathrm{H}}$ number density for all four different $\zeta^{\prime}$ simulations. For all $\zeta^{\prime}$ and for $\log _{10}\left(n_{\mathrm{H}}\right) \gtrsim 3.5$ we find very good agreement with the predicted $T_{\text {gas }}$ of B15 (their Figure 9). This is because for this range of densities, $A_{V \text {, eff }} \gtrsim 2 \mathrm{mag}$, thus the isotropic FUV is sufficiently attenuated. We note that the standard-deviation bars of $T_{\text {gas }}$ at $n_{\mathrm{H}} \gtrsim 10^{3} \mathrm{~cm}^{-3}$ decrease, while for $n_{\mathrm{H}} \sim 10^{4} \mathrm{~cm}^{-3}$ they are negligible. While the FUV has been severely extinguished, this regime is predominantly controlled by the $\mathrm{CR}$ interaction, which in turn depends very weakly on $n_{\mathrm{H}}$, as illustrated in Figure 9 of B15. We also find that the mean gas temperatures, $\left\langle T_{\text {gas }}\right\rangle_{\zeta^{\prime}}$, in each different $\zeta^{\prime}$ are $\left\langle T_{\text {gas }}\right\rangle_{1} \simeq 11 \mathrm{~K}$, $\left\langle T_{\text {gas }}\right\rangle_{10} \simeq 11 \mathrm{~K},\left\langle T_{\text {gas }}\right\rangle_{100} \simeq 22 \mathrm{~K}$ and $\left\langle T_{\text {gas }}\right\rangle_{1000} \simeq 40 \mathrm{~K}$. The low temperatures obtained for Galactic average CR energy densities are similar to those observed for FUV-shielded dark cores (see Bergin \& Tafalla 2007, for a review). Moreover, the fact that $T_{\text {gas }}$ remains low and nearly constant for modestly boosted CR energy densities (e.g., $\sim(1-10) \times$ Galactic) recovers the result obtained by Papadopoulos et al. (2011) for uniform clouds, further demonstrating the robustness of the initial conditions of star formation set deep inside such FUVshielded dense gas regions. This robustness is an important starting point for all gravoturbulent theories of star formation

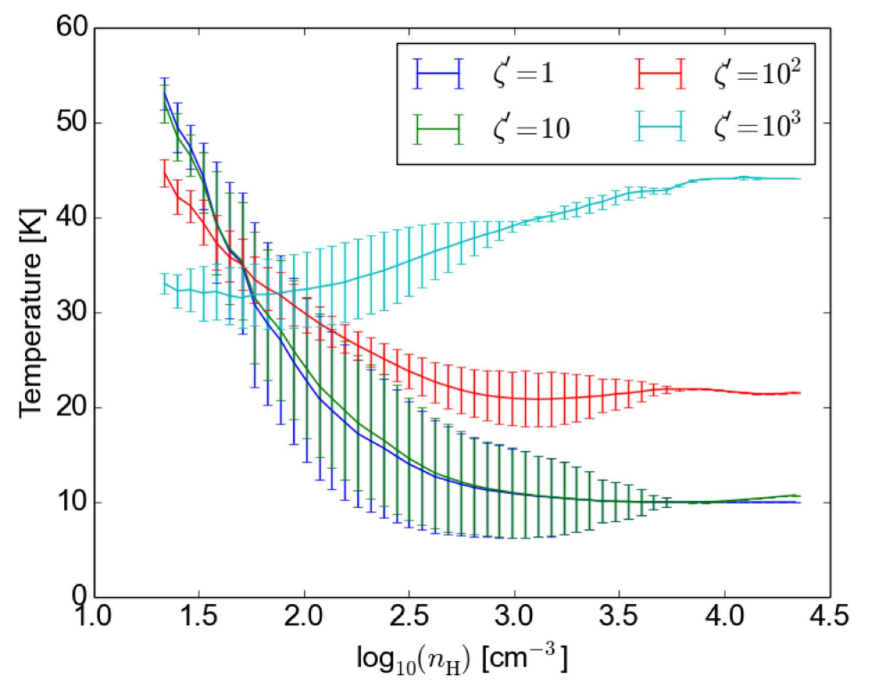

Figure 8. Correlation of $n_{\mathrm{H}}$ with the gas temperature, $T_{\mathrm{gas}}$. The error bars correspond to $1 \sigma$ standard deviation, and they are decreasing with $n_{\mathrm{H}}$ since in this regime, chemistry is primarily controlled by $\mathrm{CRs}$, which depends weakly on $n_{\mathrm{H}}$. The temperatures obtained for $n_{\mathrm{H}} \gtrsim 500 \mathrm{~cm}^{-3}$ are similar to those predicted by B 15 (their Figure 9). For low $n_{\mathrm{H}}$ (which are in principle located at low $A_{V \text {,eff }}$; see Figure 7), we find that $T_{\text {gas }}$ decreases by increasing $\zeta^{\prime}$ as a result of the increase in $\left[\mathrm{C}^{+}\right]$abundance and the associated $\mathrm{C}^{+} 158 \mu \mathrm{m}$ cooling line emission.

inside GMCs (Papadopoulos et al. 2011, and references therein).

We also note that for low densities, i.e., $n_{\mathrm{H}}<10^{2} \mathrm{~cm}^{-3}$, which are found mostly in outer cloud layers and which are in principle exposed to the isotropic FUV radiation, $T_{\text {gas }}$ decreases as $\zeta^{\prime}$ increases. This is because the FUV radiation along with the high $\mathrm{CR}$ ionization rate creates large amounts of $\mathrm{C}^{+}$, whose emission line is an effective coolant (as discussed in Section 3.2), driving the decrease of $T_{\text {gas }}$.

To further understand how the abundance distribution of species changes with $\zeta^{\prime}$, it is convenient to correlate them with $A_{V \text {,eff }}$. This is shown in Figure 9, where panels (a)-(e) show the abundances of $\mathrm{H}_{2}, \mathrm{HI}, \mathrm{C}^{+}, \mathrm{C}$, and $\mathrm{CO}$, and panel (f) shows the abundance for $T_{\text {gas }}$ versus $A_{V, \text { eff }}$. As demonstrated earlier, the abundance of $\mathrm{H}_{2}$ remains remarkably similar under all $\zeta^{\prime}$ values. The differences in $\mathrm{H}_{2}$ abundance as a function of $\zeta^{\prime}$ are reflected in the abundance of $\mathrm{H} \mathrm{I}$ in each case; here we can see that for all $\zeta^{\prime},[\mathrm{HI}] \lesssim 10^{-1}$ in the interior of the cloud, i.e., where $A_{V, \text { eff }}>7 \mathrm{mag}$. In contrast, $\mathrm{C}^{+}, \mathrm{C}$, and $\mathrm{CO}$ depend more sensitively on an increasing $\zeta^{\prime}$ with $\mathrm{CO}$ abundance destroyed even at high-density clumps close to the center of the GMC when $\zeta^{\prime}$ are high. As expected, $\mathrm{C}$ and $\mathrm{C}^{+}$follow the reverse trend, in which they increase in abundance with increasing $\zeta^{\prime}$. Observe again that for $\zeta^{\prime}=10^{3}$, the abundance of $\mathrm{C}$ is lower than in $\zeta^{\prime}=10^{2}$ (as it is also destroyed), indicating that there is a range of CR energy densities for which the overall abundance of $\mathrm{C}$ peaks and where the $\mathrm{C}$-to- $\mathrm{H}_{2}$ method will be particularly robust. We note that in both Figures 8 and 9(f), the error bars (corresponding to $1 \sigma$ standard deviation) are much smaller for high $n_{\mathrm{H}}$ and $A_{V \text {,eff }}$, respectively, meaning that $T_{\text {gas }}$ in this regime is approximately uniform and entirely controlled by CR heating.

\section{Thermal Balance and the Crucial Role of $\mathrm{OH}$}

The CO molecule can form through various channels (e.g., Herbst \& Klemperer 1973; van Dishoeck \& Black 1988; 

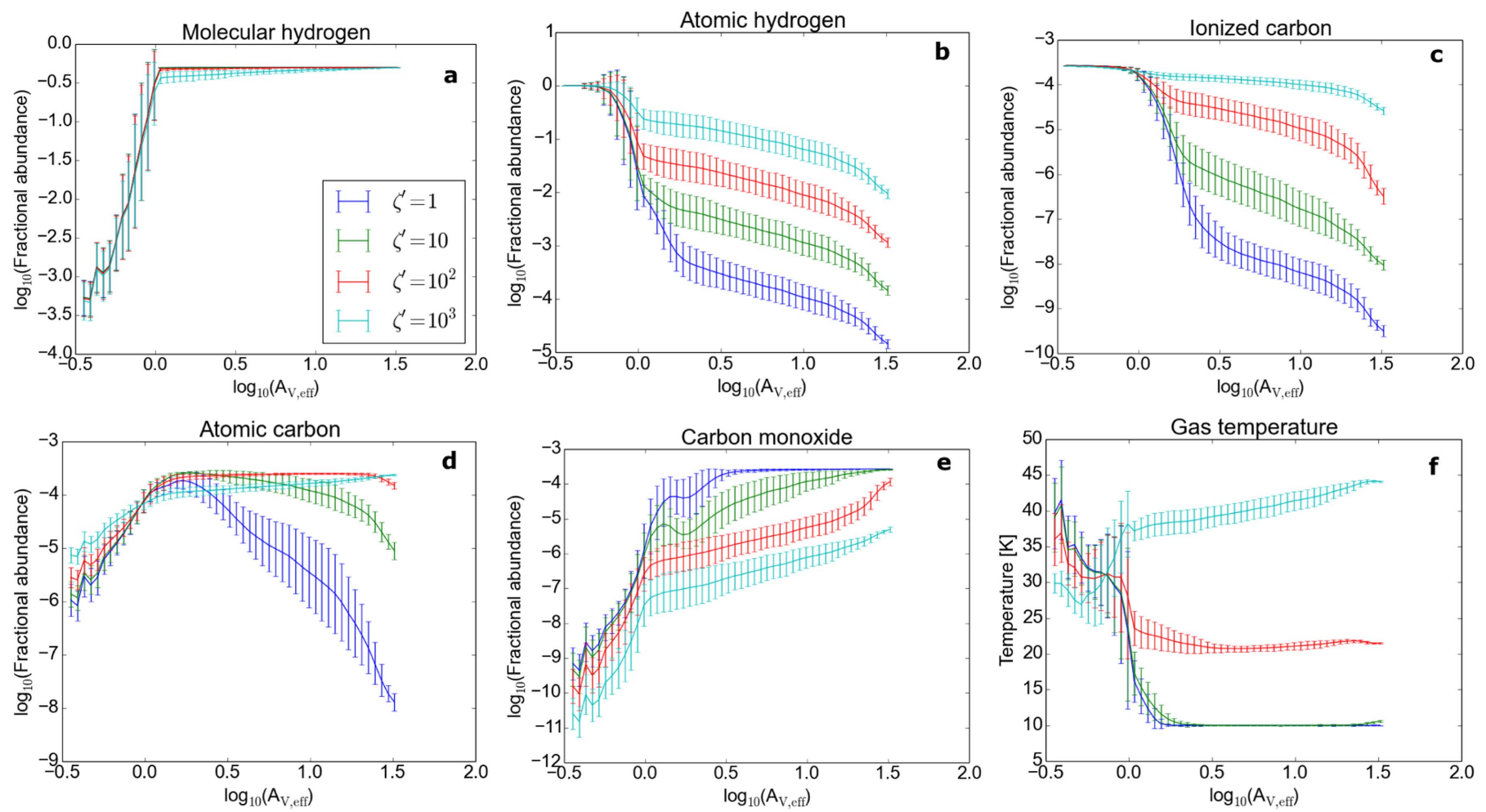

Figure 9. Correlation of $A_{V, \text { eff }}$ (see Equation (8)) with the fractional abundances of $\mathrm{H}_{2}$ (panel (a)), $\mathrm{H} \mathrm{I}(\operatorname{panel}(\mathrm{b})), \mathrm{C}^{+}$(panel (c)), $\mathrm{C}$ (panel (d)), $\mathrm{CO}$ (panel (e)), and $T_{\mathrm{gas}}$ (panel (f)) for all four different $\zeta^{\prime}$. The error bars correspond to $1 \sigma$ standard deviation, and they are decreasing at higher $A_{V \text {,eff }}$ since in this regime, chemistry is primarily controlled by CRs, which depends very weakly on $n_{\mathrm{H}}$. The $\mathrm{H}_{2}$ fractional abundance remains remarkably similar in all different $\zeta^{\prime}$, whereas CO is destroyed by $\mathrm{CR}$ particles forming $\mathrm{C}^{+}$and $\mathrm{C}$. Observe also that in panel (f) and at $\log _{10}\left(A_{V, \text { eff }}\right) \lesssim-0.3, T_{\text {gas }}$ decreases with increasing $\zeta^{\prime}$ by $\sim 10 \mathrm{~K}$ when compared to the two $\zeta^{\prime}$ extrema (see also Figure 8). It can furthermore be seen that for $\zeta^{\prime} \gtrsim 10^{2}$ the gas temperature at high visual extinction is approximately uniform and entirely controlled by heating mechanisms caused by cosmic rays.

Sternberg \& Dalgarno 1995; Tielens 2013). An important formation route, especially at moderate-to-high CR or X-ray ionization rates, as well as in low-metallicity gas (Bialy \& Sternberg 2015), depends on the OH intermediary. In cold gas $\left(T_{\text {gas }} \lesssim 100 \mathrm{~K}\right)$ the ion-molecule chemistry dominates, the $\mathrm{OH}$ formation is initiated by CR ionization of atomic oxygen or its reaction with $\mathrm{H}_{3}^{+}$, and the $\mathrm{OH}$ abundance increases with $\zeta_{\mathrm{CR}}$ (Meijerink et al. 2011). However, as discussed by Bialy \& Sternberg (2015), this trend holds only up to a critical ionization rate of $\zeta_{\mathrm{CR}, \text { crit }} \approx 10^{-14} n_{3} Z^{\prime} \mathrm{s}^{-1}$ (where $n_{3}$ is the density in units of $10^{3} \mathrm{~cm}^{-3}$ and $Z^{\prime}$ is the metallicity relative to solar). For higher $\zeta_{\mathrm{CR}}$, the $\mathrm{H}$ I-to- $\mathrm{H}_{2}$ transition occurs and the abundances of both $\mathrm{OH}$ and $\mathrm{CO}$ decrease with increasing $\zeta_{\mathrm{CR}}$.

In $\mathrm{B} 15$, it was shown that the $[\mathrm{CO}] /\left[\mathrm{H}_{2}\right]$ abundance ratio changes when varying $\zeta_{\mathrm{CR}}$ and $n_{\mathrm{H}}$ number density (their Figures 1 and 7, respectively). The chemical analysis discussed in that work (their Section 4.1) used a gas temperature obtained from full thermal balance calculations. Comparison of our isothermal models at $T_{\mathrm{gas}}=100 \mathrm{~K}$ with those of Bialy \& Sternberg (2015) showed excellent agreement. Here, we additionally consider isothermal simulations at $T_{\mathrm{gas}}=50 \mathrm{~K}$ and at $20 \mathrm{~K}$ to explore how the $[\mathrm{CO}] /\left[\mathrm{H}_{2}\right]$ ratio depends on $T_{\text {gas }}$ sensitivity, a process that was left unclear in the B15 work. We complement the latter work by examining the chemical network responsible for this behavior and what determines the $[\mathrm{CO}] /\left[\mathrm{H}_{2}\right]$ ratio at different temperatures and a given $\zeta_{\mathrm{CR}}$ and $n_{\mathrm{H}}$.

We use three different isothermal models, at $T_{\text {gas }}=100 \mathrm{~K}$, at $50 \mathrm{~K}$, and at $20 \mathrm{~K}$ gas temperatures with $\zeta^{\prime}=10^{2}$. Figure 10 shows the abundances of $\mathrm{OH}$ (upper panel) and $[\mathrm{CO}] /\left[\mathrm{H}_{2}\right]$ (lower panel) for these three different temperatures in red, green, and blue, respectively. As can be seen in the upper panel of Figure 10, at $T_{\mathrm{gas}}=20 \mathrm{~K}$ (thick blue dashed lines), the abundance of $\mathrm{OH}$ slightly increases from $\zeta_{\mathrm{CR}} / n_{\mathrm{H}} \gtrsim$ $10^{-21} \mathrm{~cm}^{3} \mathrm{~s}^{-1}$ until $\sim 8 \times 10^{-19} \mathrm{~cm}^{3} \mathrm{~s}^{-1}$, at which point $\mathrm{OH}$ strongly decreases for an increasing $\zeta_{\mathrm{CR}} / n_{\mathrm{H}}$ ratio. As soon as $T_{\mathrm{gas}}$ is increased, the abundance of $\mathrm{OH}$ also increases, which affects the $[\mathrm{CO}] /\left[\mathrm{H}_{2}\right]$ ratio. In particular, for $T_{\text {gas }}=50 \mathrm{~K}$ (thick dot-dashed lines) the abundance of $\mathrm{OH}$ keeps increasing monotonically until $\sim 2 \times 10^{-17} \mathrm{~cm}^{3} \mathrm{~s}^{-1}$, where it peaks at an abundance of $\simeq 2.5 \times 10^{-7}$ with respect to hydrogen. For $T_{\text {gas }}=100 \mathrm{~K}$ (thick red solid lines), the $\mathrm{OH}$ abundance peaks at $\simeq 3 \times 10^{-6}$. This trend is reflected in the $[\mathrm{CO}] /\left[\mathrm{H}_{2}\right]$ abundance ratio, as shown in the lower panel of this figure. In particular, for $T_{\mathrm{gas}}=20 \mathrm{~K},[\mathrm{CO}] /\left[\mathrm{H}_{2}\right]$ decreases continuously with increasing $\zeta_{\mathrm{CR}} / n_{\mathrm{H}}$. For $T_{\mathrm{gas}}=50 \mathrm{~K}$ and $100 \mathrm{~K}$, a different situation is seen: for $\zeta_{\mathrm{CR}} / n_{\mathrm{H}} \gtrsim 10^{-19} \mathrm{~cm}^{3} \mathrm{~s}^{-1}$ a turnover appears with a local minimum at $\zeta_{\mathrm{CR}} / n_{\mathrm{H}} \sim 10^{-18} \mathrm{~cm}^{3} \mathrm{~s}^{-1}$ and a local maximum at $\zeta_{\mathrm{CR}} / n_{\mathrm{H}} \sim 10^{-17} \mathrm{~cm}^{3} \mathrm{~s}^{-1}$, while for higher $\zeta_{\mathrm{CR}} / n_{\mathrm{H}},[\mathrm{CO}] /\left[\mathrm{H}_{2}\right]$ falls.

$\mathrm{CO}$ forms through the $\mathrm{OH}$ intermediary, and $\mathrm{OH}$ is initiated by two important reactions: via proton transfer,

$$
\mathrm{O}+\mathrm{H}_{3}^{+} \rightarrow \mathrm{OH}^{+}+\mathrm{H}_{2}
$$

or via charge transfer,

$$
\begin{gathered}
\mathrm{O}+\mathrm{H}^{+} \rightarrow \mathrm{O}^{+}+\mathrm{H}, \\
\mathrm{O}^{+}+\mathrm{H}_{2} \rightarrow \mathrm{OH}^{+}+\mathrm{H}
\end{gathered}
$$



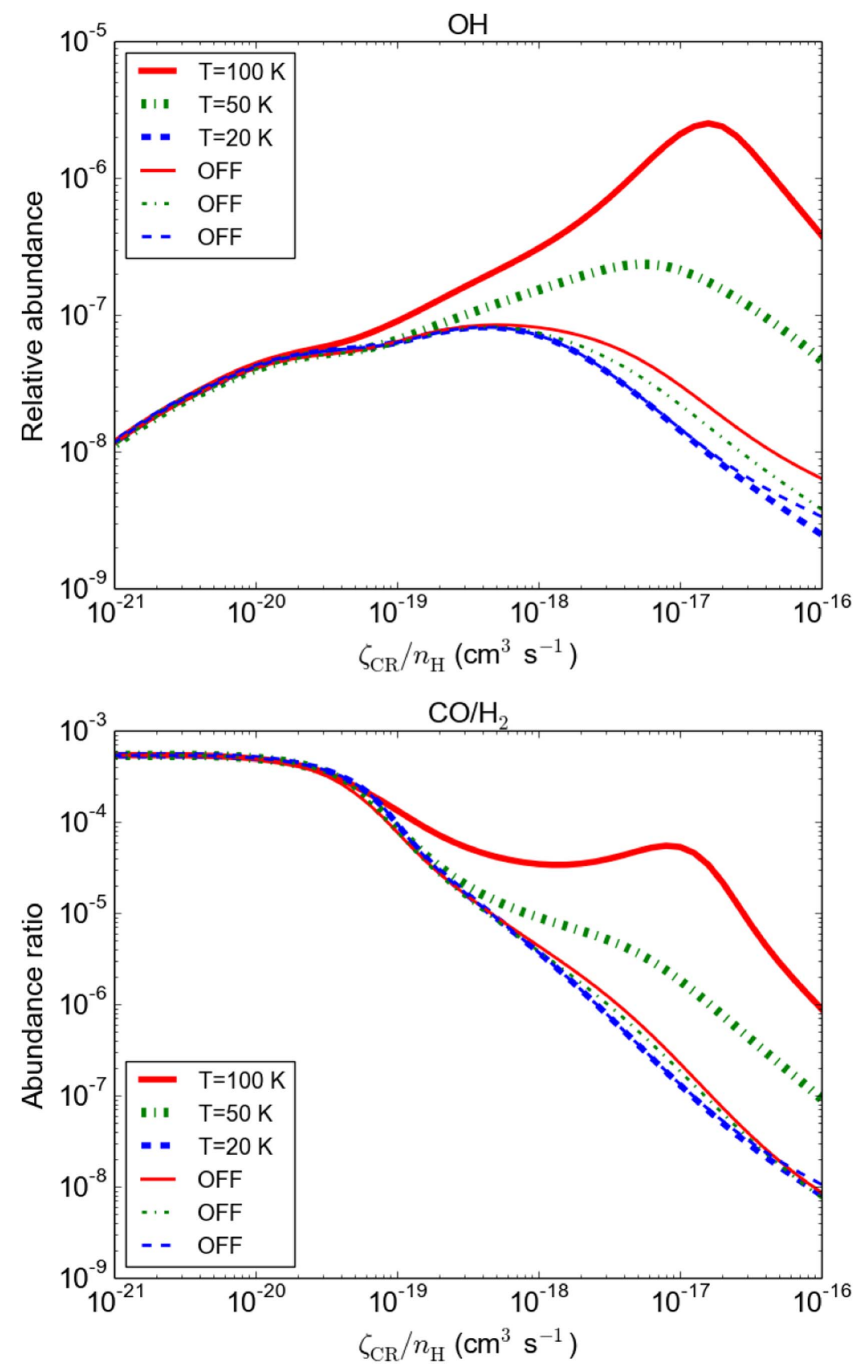

Figure 10. Isothermal test runs showing how the abundance of $\mathrm{OH}$ (top panel) relative to $\mathrm{H}$ affects the $[\mathrm{CO}] /\left[\mathrm{H}_{2}\right]$ ratio (lower panel) at different temperatures. Red (solid lines) corresponds to $T_{\text {gas }}=100 \mathrm{~K}$, green (dotted-dashed lines) to $T_{\text {gas }}=50 \mathrm{~K}$, and blue (dashed lines) to $T_{\text {gas }}=20 \mathrm{~K}$. In all cases, thick lines correspond to the case when Reaction (10) is taken into account and thin lines when it is not (OFF). It can be seen that Reaction (10) plays the dominant role controlling the $[\mathrm{CO}] /\left[\mathrm{H}_{2}\right]$ ratio at different temperatures, since it triggers the formation of $\mathrm{OH}$, which then results in the formation of $\mathrm{CO}$. By neglecting it, we do not obtain any difference in $[\mathrm{CO}] /\left[\mathrm{H}_{2}\right]$ ratio as the gas temperature increases. It thus plays a key role in determining the $\mathrm{CO}$ abundance distribution in CR-dominated regions.

(see also van Dishoeck \& Black 1986; Meijerink et al. 2011; Bialy \& Sternberg 2015). A sequence of abstraction reactions with $\mathrm{H}_{2}$ followed by dissociative recombination then leads to the formation of $\mathrm{OH}$ (see Figure 3 of Bialy \& Sternberg 2015). For low gas temperatures, Reaction (10) is substantially inefficient since it is endoergic by $224 \mathrm{~K}$ and therefore $\mathrm{OH}$ is mainly formed via the $\mathrm{H}_{3}^{+}$route (Reaction (9)). $\mathrm{CO}$ is destroyed with $\mathrm{He}^{+}$and the reaction rate increases with increasing $\zeta_{\mathrm{CR}} / n_{\mathrm{H}}$, implying that $[\mathrm{CO}] /\left[\mathrm{H}_{2}\right]$ also decreases with increasing $\zeta_{\mathrm{CR}} / n_{\mathrm{H}}$. We note that at all times, as we have illustrated above, $\mathrm{H}_{2}$ remains unaffected and all changes in the $[\mathrm{CO}] /\left[\mathrm{H}_{2}\right]$ ratio reflect mostly the $\mathrm{CO}$ behavior.

For high gas temperatures and as long as $\zeta_{\mathrm{CR}} / n_{\mathrm{H}} \lesssim 10^{-19} \mathrm{~cm}^{3} \mathrm{~s}^{-1}$, the abundance of protons is low and therefore $\mathrm{OH}$ formation is dominated by Reaction (9). This causes the abundance of $\mathrm{OH}$ at $T_{\text {gas }}=50 \mathrm{~K}$ and $100 \mathrm{~K}$ to be almost identical to that at $T_{\text {gas }}=20 \mathrm{~K}$. In this $\zeta_{\mathrm{CR}} / n_{\mathrm{H}}$ regime, we furthermore find that the removal of $\mathrm{OH}$ by $\mathrm{C}^{+}$is more efficient at low gas temperatures. Once $\zeta_{\mathrm{CR}} / n_{\mathrm{H}} \gtrsim 10^{-19} \mathrm{~cm}^{3} \mathrm{~s}^{-1}$, the abundance of protons is rapidly increasing and Reaction (10) becomes very efficient. This reflects the sudden increase in $\mathrm{OH}$ abundance (red solid line of Figure 10 upper panel) and hence $[\mathrm{CO}] /\left[\mathrm{H}_{2}\right]$ rises (red solid line, lower panel). Finally, for $\zeta_{\mathrm{CR}} / n_{\mathrm{H}} \sim 10^{-17} \mathrm{~cm}^{3} \mathrm{~s}^{-1}$, the $\mathrm{H}$ I-to- $\mathrm{H}_{2}$ transition takes place and more $\mathrm{HI}$ is formed. This causes the $\mathrm{OH}$ and consequently $\mathrm{CO}$ formation to become inefficient, and thus both these abundances decrease.

We then perform a test to study the contribution of Reaction (10) in determining the $[\mathrm{CO}] /\left[\mathrm{H}_{2}\right]$ abundance ratio at different gas temperatures. To do this, we neglect this reaction by setting its rate to a negligible value and rerun the models discussed here. The resulting abundances are plotted as dashed lines in the two panels of Figure 10 . For $T_{\text {gas }}=20 \mathrm{~K}$ the abundances of $\mathrm{OH}$ and $[\mathrm{CO}] /\left[\mathrm{H}_{2}\right]$ (blue dashed lines) are identical to the previous case (blue solid lines), indicating that the charge tranfer reaction is very inefficient at low temperatures. However, for higher temperatures, we see that Reaction (10) plays the dominant role in $\mathrm{OH}$ formation at high $\zeta_{\mathrm{CR}} / n_{\mathrm{H}}$ since it is primarily responsible for removing almost all protons; by neglecting it, we obtain the results of the $T_{\text {gas }}=20 \mathrm{~K}$ test (red dashed). In turn, this is reflected in the $[\mathrm{CO}] /\left[\mathrm{H}_{2}\right]$ (red dashed) as expected. This work considers Reaction (10) and uses it with temperature dependency. We find that Reaction (10) becomes important for gas temperatures exceeding $T_{\text {gas }} \gtrsim 20-30 \mathrm{~K}$.

Here it is important to consider that even in vigorously SF galaxies, temperatures significantly higher than $50 \mathrm{~K}$ may not be reached for most of their molecular gas mass. Thus the large CR-induced depressions of the average $[\mathrm{CO}] /\left[\mathrm{H}_{2}\right]$ abundance ratio are expected to be maintained by the $T_{\text {gas }}$-sensitive chemistry of the chemical network controlling the $\mathrm{OH}$ abundance. Indeed, as our Figure 1 shows, even when $\zeta_{\mathrm{CR}}=10^{3} \times$ Galactic (ULIRG-type of ISM), $T_{\text {gas }} \lesssim 50 \mathrm{~K}$. Furthermore, for metal-rich ISM environments, FUV photons cannot propagate through sufficiently high gas mass fractions to raise the average $T_{\text {gas }}$ beyond that range either (e.g., Papadopoulos et al. 2014), while turbulent heating can only do this for minute fractions $\lesssim 1 \%$ of molecular gas mass even in the most turbulent of clouds (Pan \& Padoan 2009; Pon et al. 2012). Exceptions to this will be places such as the Galactic Center, and possibly some very extreme ULIRGs, such as Arp 220, where $T_{\text {gas }} \sim(50-100) \mathrm{K}$ are reached, places that either do not contain much of the total $\mathrm{H}_{2}$ gas in otherwise $\mathrm{SF}$-quiescent galaxies or represent SF outliers with respect to the major mode of SF in the universe.

\section{Discussion}

In this work we recover the results of B15 of a CR-induced $\mathrm{CO}$ destruction in $\mathrm{H}_{2}$ clouds using the more realistic rendering of inhomogeneous clouds. Our three-dimensional simulations demonstrate that by increasing the $\mathrm{CR}$ ionization rate, the abundance of $\mathrm{H}_{2}$ molecule remains unaffected even for high $\mathrm{CR}$ ionization rates on the order of $10^{3}$ times the mean Galactic value. On the other hand, the $\mathrm{CO}$ abundance is sensitive to even small boosts of $\zeta_{\mathrm{CR}}$, and is easily destroyed forming $\mathrm{C}^{+}$ and consequently $\mathrm{C}$ (via recombination with free electrons) as long as gas temperatures $T_{\text {gas }} \lesssim 50 \mathrm{~K}$. Thus low- $J$ CO line emission may become very weak in such ISM environments. 
Figures 2 and 3 of B15 show that the emissivities of both $\mathrm{C}$ lines are stronger than low- $J \mathrm{CO}$ lines in $\mathrm{CO}$-poor $/ \mathrm{H}_{2}$-rich regimes. This consequently yields a potential advantage of the $\mathrm{C}$ lines in tracing the $\mathrm{CO}$-poor $\mathrm{H}_{2}$ gas around the $\mathrm{CO}$-rich regions of inhomogeneous $\mathrm{H}_{2}$ clouds (T. G. Bisbas et al. 2017, in preparation), along with the $\mathrm{CO}$-rich $\mathrm{H}_{2}$ gas. Secondary effects of a CR-induced and $n\left(\mathrm{H}_{2}\right)$-sensitive $\mathrm{CO}$ destruction can cause the visible $\mathrm{H}_{2}$ gas distributions in $\mathrm{SF}$ galaxies that are traced by the low- $J$ CO line to appear clumpier than they actually are. This has been discussed by B15, but here these effects are computed for inhomogeneous $\mathrm{H}_{2}$ clouds irradiated by elevated CR energy backgrounds (see Figure 1). It is worth noting that the CR-induced effects studied in this work mark the warm "end" of the thermal states of potentially COinvisible $\mathrm{H}_{2}$ gas, while those affected by an enhanced cosmic microwave background radiation on low- $J$ CO line (and dust continuum) brightness distributions at high redshifts mark the cold "end" (Zhang et al. 2016). Both of these regimes may contain large amounts of molecular gas in galaxies at high $(z \gtrsim 3)$ redshifts because SF is typically a highly inefficient process, i.e., there will always be large amounts of cold non-SF $\mathrm{H}_{2}$ gas and dust mass even in SF galaxies.

\subsection{Main-sequence Galaxies: on the CR “Firing” Line}

The destruction of the CO molecule by SF-powered CRs in $\mathrm{H}_{2}$-rich galaxies is of great importance for studying star formation and its modes in the early universe where such galaxies still strongly evolve. This is because the $\mathrm{H}_{2}$ gas mass surface density and $\mathrm{H}_{2}$ gas velocity dispersions are deduced from low- $J$ CO lines and are the tools to evaluate the stability of gas-rich disks via the $Q$-Toomre criterion (Daddi et al. 2010; Hodge et al. 2012). Current theoretical views of what drives SF in strongly evolving gaseous galactic disks (e.g., Elmegreen et al. 2008a, 2008b; Bournaud \& Elmegreen 2009; Bournaud et al. 2011) depend on an accurate depiction of the $\mathrm{H}_{2}$ mass surface density and velocity fields, a picture that may be incomplete because of the $\mathrm{CR}$-induced $\mathrm{CO}$ destruction in exactly such systems.

In $\mathrm{B} 15$ we discussed the possibility that the average [CO]/ $\left[\mathrm{H}_{2}\right]$ abundance may remain high in the ISM of U/LIRGs because the effect of CRs is countered by higher average molecular gas densities. However, such strong merger/ starburst systems are not the main mode of SF in the early universe. Indeed, it is in massive gas-rich galaxies, evolving along a narrow region of stellar mass $\left(M_{*}\right)$-SFR plane, the socalled MS galaxies (Noeske et al. 2007), where $\sim 90 \%$ of the cosmic star formation takes place up to $z \sim 3$ (e.g., Bell et al. 2005; Elbaz et al. 2007; Rodighiero et al. 2011). These systems, with $\mathrm{SFR} \sim(20-300) M_{\odot} \mathrm{yr}^{-1} \quad$ (e.g., Genzel et al. 2012) and seemingly ordinary GMCs (Carleton et al. 2017), are expected to be the most affected by a CR-induced destruction of CO. This is apparent from Figure 1 of B15 (where BzK galaxies are MS systems) as well as Figure 1 of this work, from which it can be seen that for $\mathrm{SFR} \sim(10-100) M_{\odot} \mathrm{yr}^{-1}$, which may correspond to $\zeta^{\prime} \sim 10-10^{2}$, the CO "marking" of a typical molecular cloud is significantly reduced.

The metallicity-insensitive CR-induced destruction of $\mathrm{CO}$ in MS galaxies can only compound the difficulties already posed by the lower metallicities prevaling in some of these systems (Genzel et al. 2012, and references therein), making provisional any $\mathrm{CO}$-deduced $\mathrm{H}_{2}$ gas mass distributions, their scale-lengths, their SFR-controlled gas depletion timescales, dynamical masses, and $Q$-Toomre stability criteria. In this context it is important to remember that even the well-known effects of strong FUV /low- $Z$ on the $[\mathrm{CO}] /\left[\mathrm{H}_{2}\right]$ abundance ratio can render entire clouds CO-free (Pak et al. 1998; Bolatto et al. 1999), which boosts their $\mathrm{C}$ (and $\mathrm{C}^{+}$) content. In the $Z-\chi_{0} / \mathrm{CR}$ domain where a phase transition to very CO-poor $\mathrm{H}_{2}$ gas phases occurs, it will be highly nonlinear, making a practical calibration of the so-called $X_{\mathrm{CO}}$ factor in MS galaxies (Daddi et al. 2010; Genzel et al. 2012; Carleton et al. 2017) and local spiral LIRGs (Papadopoulos et al. 2012a) challenging, even for their $\mathrm{CO}$-marked $\mathrm{H}_{2}$ gas distributions.

Indeed, as we have discussed in Section 3.1, the ability of $\mathrm{CO}$ to survive only in the densest regions of CR-irradiated clouds (Figure 1) can yield a misleading picture about the actual $\mathrm{H}_{2}$ gas distribution and its thermal and dynamical state. Moreover, a nearly Galactic $X_{\mathrm{CO}}$ factor may still be obtained if such CO-marked subregions of the underlying $\mathrm{H}_{2}$ distribution are used for its calibration, even as they are no longer representative of the actual $\mathrm{H}_{2}$ clouds. This can be shown if we consider the dense $\mathrm{H}_{2}$ gas regions where $\mathrm{CO}$ survives embedded in columns of $\mathrm{CO}$-free $\mathrm{H}_{2}$ gas. The latter will exert a nonthermal pressure of

$$
P_{\mathrm{e}} \approx \frac{\pi}{2} G \Sigma\left(\mathrm{H}_{2}\right)\left[\Sigma\left(\mathrm{H}_{2}\right)+\left(\frac{\sigma_{g}(V)}{\sigma_{*}(V)}\right) \Sigma_{*}\right]
$$

where we assumed that the CO-rich gas regions lie mid-plane in a rotating $\mathrm{H}_{2}$-rich disk, with stars mixed in, at a surface mass density of $\Sigma_{*}$ and vertical velocity dispersion of $\sigma_{*}(V)$ (with $\Sigma\left(\mathrm{H}_{2}\right)$ and $\sigma_{g}(V)$ the corresponding quantities for the $\mathrm{H}_{2}$ gas). For the Milky Way, $P_{\mathrm{e}} / k_{\mathrm{B}} \sim 1.4 \times 10^{4} \mathrm{~cm}^{-3} \mathrm{~K}$ is the average nonthermal pressure on the boundaries of molecular clouds (Elmegreen 1989). This crucially determines the normalization of the so-called line width-size relation for a molecular cloud of radius $R$ :

$$
\sigma(R)=\sigma_{0}\left(\frac{P_{\mathrm{e}} / k_{\mathrm{B}}}{10^{4} \mathrm{~K} \mathrm{~cm}^{-3}}\right)^{1 / 4}\left(\frac{R}{\mathrm{pc}}\right)^{1 / 2}
$$

(Chieze 1987; Elmegreen 1989). Should a CO-invisible $\mathrm{H}_{2}$ gas mass lie on top of CO-rich (and thus observable) cloud regions in the mid-plane of a SF disk, it would exert an overpressure on the CO-rich cloud regions. This would appear as a deviation from a Galactic line-width size relation and CO clouds that would seem to be out of virial equillibrium, lowering their corresponding $X_{\mathrm{CO}}$ factor (Downes \& Solomon 1998; Papadopoulos et al. 2012a). Nevertheless, the very weak dependance of the line-width size relation on $P_{\mathrm{e}}$ allows large amounts of $\mathrm{CO}$-invisible gas to exist without easily discernible observational effects. For a purely $\mathrm{H}_{2}$ gas disk $\left(\Sigma_{*}=0\right.$ ) (assumed here for simplicity), a $\Sigma_{g}(\mathrm{CO}-$ invisible $)=5 \times \Sigma_{g}(\mathrm{CO}-$ visible $)$ higher $\mathrm{H}_{2}$ gas surface density would raise $P_{\mathrm{e}}$ by a factor of 25 , but the corresponding $\sigma(R)$ of CO-rich clouds embedded inside such overlying columns of CO-invisible gas only by a factor of $\sim 2.2$. The latter is within the observational uncertainty of the $\sigma(R)$ relation in the Galaxy (e.g., Heyer \& Brunt 2004) and thus any $X_{\mathrm{CO}}$ calibration of such overpressured CO-rich clouds would still give a value consistent with a Galactic one within the uncertainties. 


\subsection{CO Chemistry in SF Galaxies: Toward a Dynamical Framework}

The physics and chemistry of the CR-induced destruction of $\mathrm{CO}$ can now be readily used in galaxy-sized/cosmological evolution models. Doing so will (a) shed light on what occurs in the context of galaxy evolution models as the $\chi_{0} / \mathrm{CR}$ boundary conditions of molecular clouds evolve, and (b) help the interface of such models with actual observables (e.g., low$J \mathrm{CO}$ and $\mathrm{C}$ images of galaxies with ALMA/JVLA). Past work has already incorporated, in a subgrid fashion, the effects of FUV destruction of $\mathrm{CO}$ in $\mathrm{H}_{2}$ clouds inside galaxies (Pelupessy \& Papadopoulos 2009; Olsen et al. 2015; Richings \& Schaye 2016). CR-driven effects will be even easier to implement in such models insofar as full transparency of $\mathrm{H}_{2}$ clouds to $\mathrm{CRs}$ and $U_{\mathrm{CR}} \propto \rho_{\mathrm{SFR}}$ are assumed. Moreover, $\mathrm{C}$ lines as $\mathrm{H}_{2}$ gas mass tracers in galaxies at high redshifts have previously been discussed in a cosmological context (Tomassetti et al. 2014). Once CR effects are taken into account in galaxy-scale models, one can then (a) evaluate the best method(s) in obtaining the $\mathrm{H}_{2}$ mass distributions and velocity fields in SF galaxies evolving across cosmic epoch, and (b) ascertain whether current theoretical views about the role of unstable giant $\mathrm{H}_{2}$ clumps in driving the $\mathrm{SF}$ of gas-rich early galaxies (Bournaud et al. 2014) still hold (e.g., a C-imaged $\Sigma\left(\mathrm{H}_{2}\right)$ distribution may be a smoother one than a $\mathrm{CO}$-imaged one in a CR-irradiated gaseous disk, impacting also the deduced gas velocity fields from these lines).

Such models can also shed light on another very important caveat discussed by B15, namely the role of turbulence. Observations of the so-called (U)LIRGs, extreme merger/ starburst systems, indicate that regions with high SFR density (and thus $\zeta_{\mathrm{CR}}$ ) are also regions of strong turbulence of $\mathrm{H}_{2}$ clouds, and thus of high $M\left(n_{\mathrm{H}}>10^{4} \mathrm{~cm}^{-3}\right) / M\left(\mathrm{H}_{2}\right)$ mass fractions per GMC. With $\mathrm{CO}$ remaining abundant in highdensity gas $\left(n_{\mathrm{H}}>10^{4} \mathrm{~cm}^{-3}\right)$, even when average $\zeta_{\mathrm{CR}}$ are high, this can diminish and even counteract the effects of CRinduced $\mathrm{CO}$ destruction in such environments as now most of the $\mathrm{H}_{2}$ gas no longer resides in the low-density regime $\left(\sim 10^{2}-10^{3} \mathrm{~cm}^{-3}\right)$ as in the MW (densities where $\mathrm{CO}$ would be CR-destroyed very effectively), but at high densities. In this regard, the $[\mathrm{CO}] /\left[\mathrm{H}_{2}\right]$ abundance ratio obtained from our simulations is of particular interest. In Figure 11 we plot this ratio versus the total $\mathrm{H}$-nucleus number density for all four different $\zeta^{\prime}$ examined. For $\zeta^{\prime} \gtrsim 10^{2}$ most of the molecular cloud gas has $[\mathrm{CO}] /\left[\mathrm{H}_{2}\right]<10^{-5}$, making it very $\mathrm{CO}$-poor. For higher densities and according to B15, this ratio would exceed $10^{-5}$ at $\zeta^{\prime}=10^{3}$ only for $n_{\mathrm{H}}>10^{4} \mathrm{~cm}^{-3}$ assuming no significant freeze-out.

Numerical simulations of individual turbulent $\mathrm{H}_{2}$ clouds study the effects of constant preset FUV radiation fields and $\mathrm{CR}$ energy densities on the $\mathrm{CO}$ and $\mathrm{C}$ distributions and the corresponding line emission (e.g., Glover \& Clark 2016, as well as the present work). Such models, while useful in finding trends of $\mathrm{CO}$ and $\mathrm{C}$ line emission as $\mathrm{H}_{2}$ gas mass tracers in GMCs, cannot address the issue of what occurs when such clouds are immersed in actual galaxies, where the FUV and CR energy densities around these clouds vary strongly on timescales equal to or shorter than internal cloud chemical and dynamical timescales (Pelupessy et al. 2006). This is because in individual cloud simulations the boundary conditions of FUV radiation, $\zeta_{\mathrm{CR}}$, and turbulent energy injection are not tracked. Galaxy-sized models (e.g., Smith et al. 2014) that

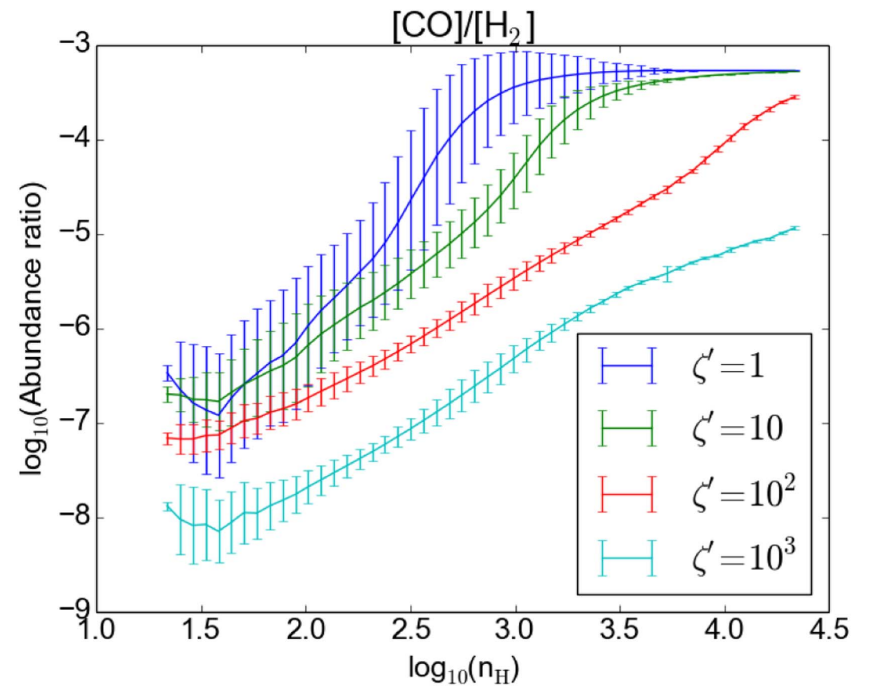

Figure 11. $[\mathrm{CO}] /\left[\mathrm{H}_{2}\right]$ abundance ratio as a function of the total $\mathrm{H}$-nucleus number density, $n_{\mathrm{H}}$, for the four different $\zeta^{\prime}$. For $\zeta^{\prime} \lesssim 10$ we do not observe any appreciable differences in the change of the ratio, whereas for $\zeta^{\prime} \gtrsim 10^{2}$ and particularly for $\zeta^{\prime} \sim 10^{3}$, almost all gas of the fractal cloud has a $[\mathrm{CO}] /$ $\left[\mathrm{H}_{2}\right]<10^{-5}$, indicating that $\mathrm{H}_{2}$ remains unaffected, but $\mathrm{CO}$ has been destroyed to form mainly $\mathrm{C}$ and $\mathrm{C}^{+}$.

include $\mathrm{H}_{2}$ clouds, along with the appropriate physics and chemistry behind the FUV/CR drivers, modeled in tandem with the evolving conditions of an SF galaxy are thus invaluable in examining whether in high SFR density environments $\mathrm{H}_{2}$ gas remains mostly CO-rich (see Pelupessy \& Papadopoulos 2009, for an early such treatment where CO and $\mathrm{H}_{2}$ are treated separately and FUV radiation onto clouds within galaxies is tracked).

Regardless of any future theoretical verdict on whether COinvisible molecular gas can exist in large quantities in SF galaxies in the early universe during periods of high SFR densities, observations of low- $J \mathrm{CO}$ and $\mathrm{C}$ lines in such systems are indispensable. Here we reiterate that the $\mathrm{C}$ column density retains its robustness in tracing $\mathrm{H}_{2}$ column near-proportionally for $\zeta_{\mathrm{CR}} \sim 10^{-15} \mathrm{~s}^{-1}$ (see Figure 1). In particular, we find that for such CR ionization rates, $N(\mathrm{C} \mathrm{I}) \simeq 4 \times 10^{-4} N\left(\mathrm{H}_{2}\right)$ (for our adopted carbon elemental abundance). This relation depends weakly on $\zeta_{\mathrm{CR}}$ provided that $\zeta_{\mathrm{CR}} \gtrsim 10^{-15} \mathrm{~s}^{-1}$, in contrast to the corresponding one for $\mathrm{CO}$. Moreover, even for the MW-level of $\zeta_{\mathrm{CR}}$ values, the $W_{\mathrm{CI}, 1-0}$ per beam will remain larger than that of CO $J=1-0$ or $J=2-1$ (the two CO transitions used to trace the bulk of $\mathrm{H}_{2}$ mass in galaxies) as long as the same beam is used to image the $\mathrm{H}_{2}$ gas in all lines. ${ }^{15}$ This, along with the possibility that $\mathrm{C}$ line imaging of $\mathrm{SF}$ disks at high- $z$ finds a different (smoother and/or possibly more extended) $\mathrm{H}_{2}$ gas distribution because of large quantities of $\mathrm{CO}$-poor $\mathrm{H}_{2}$, argues strongly for sensitive $\mathrm{C}$ line imaging of gas-rich $\mathrm{SF}$ galaxies (T. G. Bisbas et al. 2017, in preparation).

In this work we also identified the exact chemistry behind the large gas temperature sensitivity on the $\mathrm{CO}$ formation in $\mathrm{H}_{2}$ clouds (see Section 4), an issue initially discussed by B15. The

\footnotetext{
15 The lowest observed brightness temperature ratio of $W_{\mathrm{CI}, 1-0} / W_{\mathrm{CO}(1-0)}$ in the Milky Way is $\sim 0.1$ (Papadopoulos et al. 2004). If we were to observe $W_{\mathrm{CI}, 1-0}$ and $W_{\mathrm{CO}(1-0)}$ at the same resolution, then in the Rayleigh-Jeans regime the flux per beam boost would be $(492 \mathrm{GHz} / 115 \mathrm{GHz})^{2} \times 0.1 \sim 1.83$. This can lead to signal-to-noise ratio advantages for the $\mathrm{C}$ line obsrevations depending on the redshift of the object (see Papadopoulos et al. 2004). If $\zeta^{\prime}$ is increased, C lines are becoming brighter still, increasing this kind of advantage.
} 
gas temperature sensitivity of our results elevates the importance of reliable computation of the average thermal state of $\mathrm{H}_{2}$ gas in $\mathrm{FUV} / \mathrm{CR}$-intensive environments found within SF galaxies. As demonstrated in Figure 4, CRs can provide an important heating source throughout the GMC and particularly in regions with $n_{\mathrm{H}}>10^{3} \mathrm{~cm}^{-3}$. This in turn can increase the gas temperature deep in the cloud (where the FUV has been severely attenuated) to values on the order of $\sim 50 \mathrm{~K}$ (Meijerink et al. 2011; Papadopoulos et al. 2011). Such gas temperatures are still low for the $\mathrm{CO}$ formation to occur via the $\mathrm{O}+\mathrm{H}^{+}$charge transfer. In low-metallicity galaxies, a higher gas temperature may be expected as cooling efficiency and shielding is lower than in solar metallicity galaxies, perhaps moderating the CR-induced destruction of $\mathrm{CO}$ in high $\zeta^{\prime}$ environments, as discussed in Section 4. It is thus necessary that the CR effects are studied together with those driven by lower metallicities in order to discern their combined impact on the average $[\mathrm{CO}] /\left[\mathrm{H}_{2}\right]$ abundance in metal-poor SF galaxies.

Even though we used standard cooling/heating mechanisms of PDR/CRDR physics (see Section 3.2), turbulence will also heat the molecular gas (e.g., Pan \& Padoan 2009), and do so in a volumetric manner just like the CRs. Turbulent heating has even been argued as a dominant heating mechanism of galaxysized $\mathrm{H}_{2}$ gas reservoirs in some extreme $\mathrm{SF}$ galaxies (Papadopoulos et al. 2012b), even as numerical simulations of individual molecular clouds show that turbulent heating typically affects only $\lesssim 1 \%$ of their mass (Pan \& Padoan 2009; Pon et al. 2012). The so-called Brick cloud is a well-studied object close to the Galactic Centre. Simulations performed by Clark et al. (2013) have reproduced its observed gas and dust temperatures when their modeled cloud interacts with a FUV of strength $\chi / \chi_{0} \sim 10^{3}$ and a $\zeta_{\mathrm{CR}} \sim 10^{-14} \mathrm{~s}^{-1}$. Early suggestions by Ao et al. (2013) proposed that the gas heating of the CMZ at the Galactic Centre is primarily dominated by CRs and/or turbulence. Recent observations by Ginsburg et al. (2016), however, show that the dominant heating mechanism of the particular Brick cloud is turbulence, which, in association with an LVG analysis, gives an upper limit of $\zeta_{\mathrm{CR}}<10^{-14} \mathrm{~s}^{-1}$. However, in places of the CMZ, CRs may still be a very important heating source, particular in less turbulent subregions (Ginsburg et al. 2016).

Our simple treatment of turbulent heating (Black 1987; Rodríguez-Fernández et al. 2001; Bisbas et al. 2012) leaves unaswered the question of how much it can influence the average thermal states of the typically very turbulent $\mathrm{H}_{2}$ gas in extreme starbursts with high SFR densities. Numerical simulations of individual $\mathrm{H}_{2}$ gas clouds, at Mach numbers appropriate for the ISM of galaxies with very high SFR densities $(\mathcal{M} \sim 3-10$ times that of ordinary spirals), which include turbulent heating along with the chemistry and physics of CR-induced $\mathrm{CO}$ destruction are necessary to answer this question. If strong turbulence can elevate the average $\mathrm{H}_{2}$ gas temperatures and densities of galaxies with high SFR densities (typically merger/starbursts), it may still keep the $[\mathrm{CO}] /\left[\mathrm{H}_{2}\right]$ abundance ratio high and the $\mathrm{H}_{2}$ gas traceable via the traditional methods based on CO (see B15 for the relevant discussion).

\section{Conclusions}

In this paper, continuing the study of Bisbas et al. (2015), we present results from a suite of three-dimensional astrochemical simulations of inhomogeneous molecular clouds, rendered as a fractal, and embedded in different $\mathrm{CR}$ ionization rates spanning three orders of magnitude $\left(\zeta_{\mathrm{CR}}=10^{-17}-10^{-14} \mathrm{~s}^{-1}\right)$ along with a constant isotropic FUV radiation field $\left(\chi / \chi_{0}=1\right)$. Our study therefore focuses only on the effect of high CR ionization rates expected in SF galaxies in the universe and on how it affects the abundances of $\mathrm{CO}, \mathrm{C}, \mathrm{C}^{+}, \mathrm{H} \mathrm{I}$, and $\mathrm{H}_{2}$. We used the 3D-PDR (Bisbas et al. 2012) code to perform full thermal balance and chemistry calculations. Our results can be summarized as follows.

1. The column density and total $\mathrm{H}_{2}$ mass of a typical inhomogeneous GMC remains nearly constant for increasing $\zeta^{\prime}$, with the total mass of $\mathrm{H}_{2}$ decreasing by $\lesssim 10 \%$ for $\zeta_{\mathrm{CR}} \sim 10^{-14} \mathrm{~s}^{-1}\left(\sim 10^{3} \times\right.$ Galactic $)$. On the other hand, a significant reduction of the $[\mathrm{CO}] /\left[\mathrm{H}_{2}\right]$ abundance ratio sets in throughout the cloud, even when $\zeta_{\mathrm{CR}} \sim 10^{-16} \mathrm{~s}^{-1}(\sim 10 \times$ Galactic $)$, a value expected for the ISM of many SF galaxies in the universe.

2. When the average $\zeta_{\mathrm{CR}}$ increases further, up to $\sim 10^{-15}-10^{-14} \mathrm{~s}^{-1}$, the $\mathrm{CO}$ molecule is destroyed so thoroughly that only the densest regions of the GMC remain CO-rich. The abundances of $\mathrm{C}$ and $\mathrm{C}^{+}$, on the other hand, increase, with the latter becoming particularly abundant for $\zeta_{\mathrm{CR}} \sim 10^{-14} \mathrm{~s}^{-1}$. Atomic carbon is the species that proves to be the most abundant, marking most of the $\mathrm{H}_{2}$ mass of the cloud over a wide range of $\zeta_{\mathrm{CR}}$ values. When only $\mathrm{CO}$ rotational transitions are used to discern the average state and mass of such CR-irradiated GMCs, only their highest density peaks $\left(n_{\mathrm{H}} \gtrsim 10^{3} \mathrm{~cm}^{-3}\right)$ will be recovered, which will cause the clouds to appear clumpier than they truly are and convey biased information on the molecular gas velocity fields.

3. We expect significant effects of CR-induced destruction of CO to occur in the so-called MS Galaxies, the systems where most of the cosmic history of SF unfolds. This is a result of their high SF rates (implying high CR rates) and seemingly Galactic-type molecular clouds. The widespread CR destruction of $\mathrm{CO}$ expected in such systems will make the calibration of their $X_{\mathrm{CO}}$ factor challenging, even for their CO-bright gas.

4. Our computations recover gas temperatures of $T_{\text {gas }} \sim 10 \mathrm{~K}$ for the CR-irradiated and FUV-shielded dense regions inside these GMCs. This is indeed typical for such regions in the Galaxy, and it remains robust over $\zeta_{\mathrm{CR}} \lesssim 10^{-16} \mathrm{~s}^{-1}$. This is of particular importance if the initial conditions of SF, and the stellar initial mass function (IMF) mass scale (i.e., the IMF "knee") are indeed set within such regions. Nevertheless, once $\zeta_{\mathrm{CR}} \sim 10^{-15}-10^{-14} \mathrm{~s}^{-1}$, the temperature of such regions rises up to $T_{\text {gas }} \sim 30-50 \mathrm{~K}$, and the initial conditions of $\mathrm{SF}$ in such galaxies are bound to change.

5. The main heating mechanisms in CRDRs apart from CRs are the chemical mechanism (due to the large amounts of ions expected in CRDRs) and the $\mathrm{H}_{2}$ formation mechanism. Cooling, on the other hand, is mainly due to $\mathrm{C}^{+}$and $\mathrm{O}$, with the contribution of $\mathrm{CO}$ cooling nearly negligible, as its abundance is at least two orders of magnitude lower than in normal Galactic conditions.

6. We find the CR-regulated $[\mathrm{CO}] /\left[\mathrm{H}_{2}\right]$ abundance ratio to be sensitive to the temperature of the gas once $T_{\text {gas }}>50 \mathrm{~K}$. A significant production of the $\mathrm{OH}$ molecule, acting as an intermediary, is the $T_{\text {gas }}$-sensitive 
part of the chemical network that determines the [CO]/ $\left[\mathrm{H}_{2}\right]$ ratio. For warm gas at $T_{\text {gas }}=100 \mathrm{~K}$ abundant $\mathrm{OH}$ can keep the molecular gas CO-rich (i.e., [CO]/ $\left[\mathrm{H}_{2}\right] \sim 10^{-4}$ ), even in high $\mathrm{CR}$ energy environments. The severe CR-induced destruction of $\mathrm{CO}$ sets in for $T_{\text {gas }} \lesssim 50 \mathrm{~K}$, which our thermochemical calculations indicate as containing the bulk of $\mathrm{H}_{2}$ mass in our inhomogeneous cloud models and indeed the bulk of molecular gas in SF galaxies, except perhaps in the most extreme merger/starbursts.

7. Our simple treatment of turbulent heating, and the fact that GMCs in the very high SFR density environments of merger/starburst galaxies are much more turbulent and thus denser, necessitate careful considerations of turbulent heating and a dynamic rendering of density inhomogeneities in order to explore our findings in a fully realistic setting.

8. Finally, the chemistry and thermal-balance calculations behind the CR-controlled $[\mathrm{CO}] /\left[\mathrm{H}_{2}\right],[\mathrm{C}] /\left[\mathrm{H}_{2}\right]$, and $\left[\mathrm{C}^{+}\right] /\left[\mathrm{H}_{2}\right]$ abundance ratios inside inhomogeneous $\mathrm{H}_{2}$ clouds can be used in a subgrid fashion as elements of galaxy-sized numerical simulations of evolving galaxies. This is perhaps a vital ingredient of any realistic galaxy evolution model across cosmic epoch, given the elevated SFR densities - and thus CR energy densities - typically observed in galaxies in the distant universe.

As a final conclusion, we mention that because of the strong effects of CRs on the $\mathrm{CO}$ abundance combined with the effects of high FUV and/or low-metallicity environments in further reducing its abundance, and the impracticallity of $\mathrm{C}^{+}$imaging in SF galaxies except for the highest redshift objects $(z \gtrsim 4)$, a concerted effort must be mounted by the extragalactic community toward $\mathrm{C}$ line imaging of $\mathrm{H}_{2}$ gas in the universe as a viable alternative.

The authors thank an anonymous referee for reviewing the manuscript. The referee's comments have impoved the clarity of this work. We thank Andreas Schruba, Andrew Strong, Rob Ivison, Nick Indriolo, Steffi Walch, and Paola Caselli for the useful discussions. This work is supported by a Royal Netherlands Academy of Arts and Sciences (KNAW) professor prize, and by the Netherlands Research School for Astronomy (NOVA). The work of P.P.P. was funded by an Ernest Rutherford Fellowship. S.B. acknowledges support from the DFG via German-Israel Project Cooperation grant STE1869/21 GE625/17-1. L.Sz. acknowledges support from the A-ERC grant 108477 PALs. Z.Y.Z. acknowledges support from ERC in the form of the Advanced Investigator Programme, 321302 COSMICISM.

\section{Appendix A}

\section{Chemical Network and Initial Elemental Abundances}

We explore the dependence of our results on the choice of chemical network and the choice of initial elemental abundances. To do this, we perform a suite of OD calculations where we switch off the UV radiation field. We use densities of $n_{\mathrm{H}}=10^{2-4} \mathrm{~cm}^{-3}$ interacting with $\zeta^{\prime}=10^{0-3} \mathrm{CR}$ ionization rates (see Equation (1)). We consider two subsets and the full UMIST 2012 (McElroy et al. 2013), consisting of 33 species (4 elements: $\mathrm{H}, \mathrm{He}, \mathrm{C}$, and $\mathrm{O}$ ), 58 species (2 additional elements: $\mathrm{Mg}$ and $\mathrm{S}$ ), and 215 species (4 additional elements:
$\mathrm{Na}, \mathrm{Fe}, \mathrm{Si}$, and $\mathrm{N}$ ). In addition to Table 1, the initial abundances of the last four elements used are $\mathrm{Na}=$ $1.738 \times 10^{-6}, \mathrm{Fe}=3.162 \times 10^{-5}, \mathrm{Si}=3.236 \times 10^{-5}$, and $\mathrm{N}=6.76 \times 10^{-5}$ (Asplund et al. 2009). The results of the above tests are shown as red solid (33 species), green solid (58 species), and blue solid (215 species) lines in Figure 12.

We furthermore perform additional simulations using the subset of 58 species only, in which we change the initial values of elemental abundances to those that have been measured by optical/UV absorption lines in diffuse clouds with densities similar to those of our fractal GMCs. We use $\mathrm{C}=1.4 \times$ $10^{-4}$ (Cardelli et al. 1996), $\mathrm{O}=2.8 \times 10^{-4}$ (Cartledge et al. 2004), and $\mathrm{Mg}=7 \times 10^{-9}$ while keeping $\mathrm{S}$ as shown in Table 1 because it is observed to remain largely undepleted. The reduction of the $\mathrm{Mg}$ abundance by $\sim 4$ orders of magnitude compared to the value shown in Table 1 is motivated by the fact that such high abundances of $\mathrm{Mg}$ may act as a nonnegligible source of electrons, which can in turn affect the $[\mathrm{CO}] /\left[\mathrm{H}_{2}\right]$ ratio. The results of this test are shown in Figure 12 as green dashed lines. These abundances correspond to environments with metallicities $Z \simeq Z_{\odot}$. We note that thoughout this work we have assumed solar metallicity at all times, and we do not explore the effect of CR-induced $\mathrm{CO}$ destruction in sub-solar and super-solar environments. For all reasonable assumptions, $\mathrm{C} / \mathrm{O} \sim 0.5$, which is consistent with diffuse ISM observations, and always $<1$.

Overall, from the above suite of tests, we find that the general trend of the $[\mathrm{CO}] /\left[\mathrm{H}_{2}\right]$ abundance ratio decrease by increasing the $\mathrm{CR}$ ionization rate remains robust. We therefore demonstrate that the validity of findings presented in this work and in particular the column density maps shown in Figure 1 do not strongly depend on the complexity of the chemical network used or the choice of initial elemental abundances adopted.

\section{Appendix B Mapping SPH to Grid}

We convert the properties of the cloud (number density distribution, gas temperatures, etc.) from SPH into a uniform grid in order to produce the column density plots of Figure 1. Each SPH particle, $p$, comprising the cloud has a smoothing length $h_{p}$ and carries the corresponding PDR information from the 3D-PDR calculations. In order to weight an SPH quantity, $A_{p}$, in the centroid, $q$, of a given cell of the uniform grid, we use the equation

$$
A_{q}=\sum_{p=1}^{N} n_{p} A_{p} W\left(\left|\boldsymbol{r}_{\boldsymbol{q}}-\boldsymbol{r}_{\boldsymbol{p}}\right|, h_{p}\right),
$$

where $N=50$ is the number of the closest neighboring SPH particles to the centroid of the cell and $W$ is the Monaghan \& Lattanzio (1985) softening kernel

$$
W(\ell, p)=\frac{1}{\pi h_{p}^{3}}\left\{\begin{array}{lc}
1-\frac{3}{2} \ell^{2}+\frac{3}{4} \ell^{3}, & \text { if } 0 \leqslant \ell<1 \\
\frac{1}{4}(2-\ell)^{3}, & \text { if } 1 \leqslant \ell \leqslant 2 \\
0, & \text { if } \ell>0,
\end{array}\right.
$$

where $\ell=\left|\boldsymbol{r}_{q}-\boldsymbol{r}_{p}\right| / h_{p}$. The number of SPH particles in each grid cell varies from a few tens (highest density regions) to none (outside the cloud). Similar techniques have been discussed by Price (2007). 

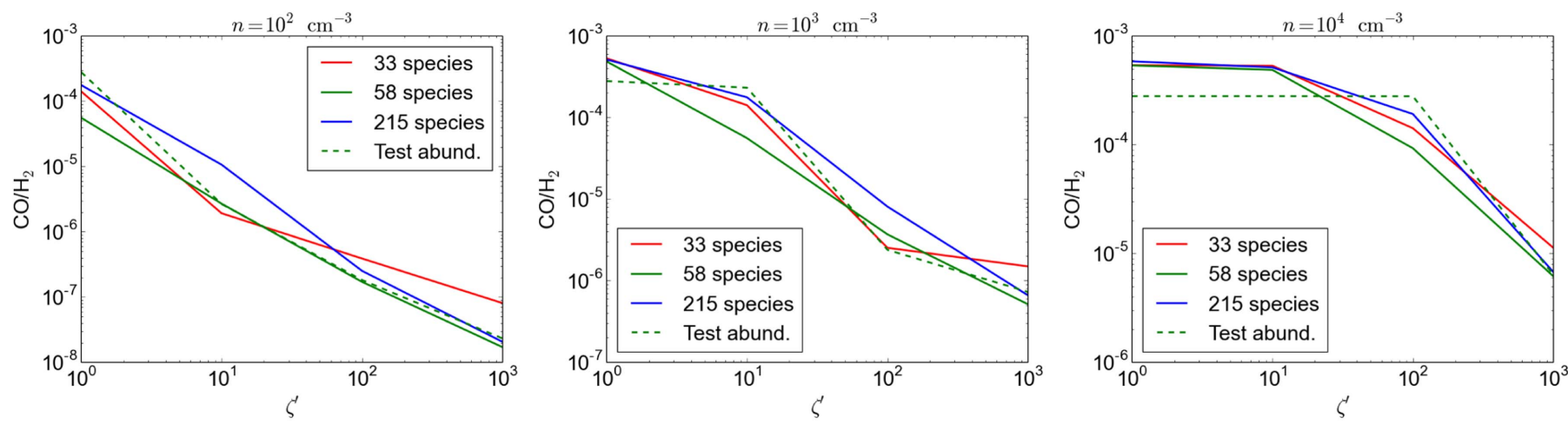

Figure 12. Dependency of the $[\mathrm{CO}] /\left[\mathrm{H}_{2}\right]$ abundance ratio on $\zeta^{\prime}$ for $n_{\mathrm{H}}=10^{2} \mathrm{~cm}^{-3}$ (left), $10^{3} \mathrm{~cm}^{-3}$ (middle), and $10^{4} \mathrm{~cm}^{-3}$ (right) using a 33 -species (red solid line), 58-species (green solid line), and 215-species (blue solid line) chemical network with initial abundances as discussed in Asplund et al. (2009). The green dashed line ("Test abund.") corresponds to the 58-species network with initial elemental abundances as discussed in Appendix A. We find that the general trend of $[\mathrm{CO}] /\left[\mathrm{H}_{2}\right]$ decrease by the increase of cosmic-ray ionization rate remains robust, supporting the validity of our results.

\section{References}

Ao, Y., Henkel, C., Menten, K. M., et al. 2013, A\&A, 550, A135 Asplund, M., Grevesse, N., Sauval, A. J., \& Scott, P. 2009, ARA\&A, 47, 481 Bakes, E. L. O., \& Tielens, A. G. G. M. 1994, ApJ, 427, 822 Bell, E. F., Papovich, C., Wolf, C., et al. 2005, ApJ, 625, 23

Bell, T. A., Roueff, E., Viti, S., \& Williams, D. A. 2006, MNRAS, 371, 1865 Bell, T. A., Viti, S., \& Williams, D. A. 2007, MNRAS, 378, 983

Bergin, E. A., \& Tafalla, M. 2007, ARA\&A, 45, 339

Bialy, S., \& Sternberg, A. 2015, MNRAS, 450, 4424

Bialy, S., \& Sternberg, A. 2016, ApJ, 822, 83

Bisbas, T. G., Bell, T. A., Viti, S., Yates, J., \& Barlow, M. J. 2012, MNRAS, 427, 2100

Bisbas, T. G., Papadopoulos, P. P., \& Viti, S. 2015, ApJ, 803, 37 (B15)

Black, J. H. 1987, in Interstellar Processes, ed. D. J. Hollenbach \& H. A. Thronson, Jr. (Dordrecht: Reidel), 731

Bolatto, A. D., Jackson, J. M., \& Ingalls, J. G. 1999, ApJ, 513, 275

Bolatto, A. D., Wolfire, M., \& Leroy, A. K. 2013, ARA\&A, 51, 207

Bothwell, M. S., Aguirre, J. E., Aravena, M., et al. 2017, MNRAS, 466, 2825

Bournaud, F., Chapon, D., Teyssier, R., et al. 2011, ApJ, 730, 4

Bournaud, F., \& Elmegreen, B. G. 2009, ApJL, 694, L158

Bournaud, F., Perret, V., Renaud, F., et al. 2014, ApJ, 780, 57

Bradford, C. M., Nikola, T., Stacey, G. J., et al. 2003, ApJ, 586, 891

Bryant, P. M., \& Scoville, N. Z. 1996, ApJ, 457, 678

Burke, J. R., \& Hollenbach, D. J. 1983, ApJ, 265, 223

Cardelli, J. A., Meyer, D. M., Jura, M., \& Savage, B. D. 1996, ApJ, 467, 334 Carleton, T., Cooper, M. C., Bolatto, A. D., et al. 2017, MNRAS, 467, 4886

Cartledge, S. I. B., Lauroesch, J. T., Meyer, D. M., \& Sofia, U. J. 2004, ApJ, 613, 1037

Cazaux, S., \& Spaans, M. 2004, ApJ, 611, 40

Cazaux, S., \& Tielens, A. G. G. M. 2002, ApJL, 575, L29

Chen, B.-Q., Liu, X.-W., Yuan, H.-B., Huang, Y., \& Xiang, M.-S. 2015, MNRAS, 448, 2187

Chieze, J. P. 1987, A\&A, 171, 225

Clark, P. C., Glover, S. C. O., \& Klessen, R. S. 2012, MNRAS, 420, 745

Clark, P. C., Glover, S. C. O., Ragan, S. E., Shetty, R., \& Klessen, R. S. 2013, ApJL, 768, L34

Clavel, J., Viala, Y. P., \& Bel, N. 1978, A\&A, 65, 435

Cummings, A. C., Stone, E. C., Heikkila, B. C., et al. 2015, ICRC, 34, 318

Daddi, E., Bournaud, F., Walter, F., et al. 2010, ApJ, 713, 686

Dalgarno, A. 2006, PNAS, 103, 12269

Dickman, R. L., Snell, R. L., \& Schloerb, F. P. 1986, ApJ, 309, 326

Downes, D., \& Solomon, P. M. 1998, ApJ, 507, 615

Draine, B. T. 1978, ApJS, 36, 595

Elbaz, D., Daddi, E., Le Borgne, D., et al. 2007, A\&A, 468, 33

Elmegreen, B. G. 1989, ApJ, 338, 178

Elmegreen, B. G., Bournaud, F., \& Elmegreen, D. M. 2008a, ApJ, 688, 67

Elmegreen, B. G., Bournaud, F., \& Elmegreen, D. M. 2008b, ApJ, 684, 829

Genzel, R., Tacconi, L. J., Combes, F., et al. 2012, ApJ, 746, 69

Genzel, R., Tacconi, L. J., Lutz, D., et al. 2015, ApJ, 800, 20

Gerin, M., \& Phillips, T. G. 2000, ApJ, 537, 644

Ginsburg, A., Henkel, C., Ao, Y., et al. 2016, A\&A, 586, A50

Glover, S. C. O., \& Clark, P. C. 2012, MNRAS, 421, 9

Glover, S. C. O., \& Clark, P. C. 2016, MNRAS, 456, 3596
Glover, S. C. O., Clark, P. C., Micic, M., \& Molina, F. 2015, MNRAS, 448, 1607

Glover, S. C. O., Federrath, C., Mac Low, M.-M., \& Klessen, R. S. 2010, MNRAS, 404, 2

Górski, K. M., Hivon, E., Banday, A. J., et al. 2005, ApJ, 622, 759

Gratier, P., Braine, J., Schuster, K., et al. 2017, A\&A, 600, A27

Gullberg, B., Lehnert, M. D., De Breuck, C., et al. 2016, A\&A, 591, A73

Habing, H. J. 1968, BAN, 19, 421

Herbst, E., \& Klemperer, W. 1973, ApJ, 185, 505

Heyer, M. H., \& Brunt, C. M. 2004, ApJL, 615, L45

Hodge, J. A., Carilli, C. L., Walter, F., et al. 2012, ApJ, 760, 11

Hollenbach, D., \& McKee, C. F. 1979, ApJS, 41, 555

Hollenbach, D. J., Takahashi, T., \& Tielens, A. G. G. M. 1991, ApJ, 377, 192

Hopkins, A. M., \& Beacom, J. F. 2006, ApJ, 651, 142

Hubber, D. A., Batty, C. P., McLeod, A., \& Whitworth, A. P. 2011, A\&A, 529, A27

Indriolo, N., \& McCall, B. J. 2012, ApJ, 745, 91

Indriolo, N., Neufeld, D. A., Gerin, M., et al. 2015, ApJ, 800, 40

Israel, F. P., \& Baas, F. 2001, A\&A, 371, 433

Krips, M., Martín, S., Sakamoto, K., et al. 2016, A\&A, 592, L3

Lacy, J. H., Knacke, R., Geballe, T. R., \& Tokunaga, A. T. 1994, ApJL, 428, L69

Lada, E. A., \& Blitz, L. 1988, ApJL, 326, L69

Le Petit, F., Ruaud, M., Bron, E., et al. 2016, A\&A, 585, A105

Lo, N., Cunningham, M. R., Jones, P. A., et al. 2014, ApJL, 797, L17

Mashian, N., Sternberg, A., \& Loeb, A. 2013, MNRAS, 435, 2407

McCall, B. J., Huneycutt, A. J., Saykally, R. J., et al. 2003, Natur, 422, 500

McElroy, D., Walsh, C., Markwick, A. J., et al. 2013, A\&A, 550, A36

Meijerink, R., Spaans, M., Loenen, A. F., \& van der Werf, P. P. 2011, A\&A, 525, A119

Monaghan, J. J., \& Lattanzio, J. C. 1985, A\&A, 149, 135

Narayanan, D., \& Krumholz, M. 2017, MNRAS, 467, 50

Neufeld, D. A., González-Alfonso, E., Melnick, G., et al. 2010, A\&A, 521, L5

Nishimura, A., Tokuda, K., Kimura, K., et al. 2015, ApJS, 216, 18

Noeske, K. G., Weiner, B. J., Faber, S. M., et al. 2007, ApJL, 660, L43

Offner, S. S. R., Bisbas, T. G., Bell, T. A., \& Viti, S. 2014, MNRAS, 440, L81

Offner, S. S. R., Bisbas, T. G., Viti, S., \& Bell, T. A. 2013, ApJ, 770, 49

Olsen, K. P., Greve, T. R., Narayanan, D., et al. 2015, ApJ, 814, 76

Padoan, P., Juvela, M., Kritsuk, A., \& Norman, M. L. 2009, ApJL, 707, L153

Padovani, M., Hennebelle, P., \& Galli, D. 2013, A\&A, 560, A114

Pak, S., Jaffe, D. T., van Dishoeck, E. F., Johansson, L. E. B., \& Booth, R. S. 1998, ApJ, 498, 735

Pan, L., \& Padoan, P. 2009, ApJ, 692, 594

Papadopoulos, P. P. 2010, ApJ, 720, 226

Papadopoulos, P. P., \& Greve, T. R. 2004, ApJL, 615, L29

Papadopoulos, P. P., \& Seaquist, E. R. 1999, ApJ, 516, 114

Papadopoulos, P. P., Thi, W.-F., Miniati, F., \& Viti, S. 2011, MNRAS, 414, 1705

Papadopoulos, P. P., Thi, W.-F., \& Viti, S. 2002, ApJ, 579, 270

Papadopoulos, P. P., Thi, W.-F., \& Viti, S. 2004, MNRAS, 351, 147

Papadopoulos, P. P., van der Werf, P., Xilouris, E., Isaak, K. G., \& Gao, Y. 2012a, ApJ, 751, 10

Papadopoulos, P. P., van der Werf, P. P., Xilouris, E. M., et al. 2012b, MNRAS, 426, 2601

Papadopoulos, P. P., Zhang, Z.-Y., Xilouris, E. M., et al. 2014, ApJ, 788, 153 
Pelupessy, F. I., \& Papadopoulos, P. P. 2009, ApJ, 707, 954

Pelupessy, F. I., Papadopoulos, P. P., \& van der Werf, P. 2006, ApJ, 645, 1024

Planck Collaboration, Ade, P. A. R., Aghanim, N., et al. 2016, A\&A, 594, A28

Polychroni, D., Moore, T. J. T., \& Allsopp, J. 2012, MNRAS, 422, 2992

Pon, A., Johnstone, D., \& Kaufman, M. J. 2012, ApJ, 748, 25

Price, D. J. 2007, PASA, 24, 159

Richings, A. J., \& Schaye, J. 2016, MNRAS, 460, 2297

Rimmer, P. B., Herbst, E., Morata, O., \& Roueff, E. 2012, A\&A, 537, A7

Rodighiero, G., Daddi, E., Baronchelli, I., et al. 2011, ApJL, 739, L40

Rodríguez-Fernández, N. J., Martín-Pintado, J., Fuente, A., et al. 2001, A\&A, 365,174

Rowan-Robinson, M. 1980, ApJS, 44, 403

Sánchez, N., Añez, N., Alfaro, E. J., \& Crone Odekon, M. 2010, ApJ, 720, 541

Sanders, D. B., Mazzarella, J. M., Kim, D.-C., Surace, J. A., \& Soifer, B. T. 2003, AJ, 126, 1607

Schöier, F. L., van der Tak, F. F. S., van Dishoeck, E. F., \& Black, J. H. 2005 , A\&A, 432, 369

Smith, R. J., Glover, S. C. O., Clark, P. C., Klessen, R. S., \& Springel, V. 2014, MNRAS, 441, 1628

Solomon, P. M., Downes, D., Radford, S. J. E., \& Barrett, J. W. 1997, ApJ, 478,144

Solomon, P. M., Rivolo, A. R., Barrett, J., \& Yahil, A. 1987, ApJ, 319, 730

Sternberg, A., \& Dalgarno, A. 1995, ApJS, 99, 565
Sternberg, A., Le Petit, F., Roueff, E., \& Le Bourlot, J. 2014, ApJ, 790, 10 Strong, A. W., Moskalenko, I. V., \& Reimer, O. 2004a, ApJ, 613, 962

Strong, A. W., Moskalenko, I. V., Reimer, O., Digel, S., \& Diehl, R. 2004b, A\&A, 422, L47

Szúcs, L., Glover, S. C. O., \& Klessen, R. S. 2016, MNRAS, 460, 82

Tielens, A. G. G. M. 2013, RvMP, 85, 1021

Tielens, A. G. G. M., \& Hollenbach, D. 1985, ApJ, 291, 722

Tomassetti, M., Porciani, C., Romano-Díaz, E., Ludlow, A. D., \& Papadopoulos, P. P. 2014, MNRAS, 445, L124

van Dishoeck, E. F. 1992, in IAU Symp. 150, Astrochemistry of Cosmic Phenomena, ed. P. D. Singh (Dordrecht: Kluwer), 143

van Dishoeck, E. F., \& Black, J. H. 1986, ApJS, 62, 109

van Dishoeck, E. F., \& Black, J. H. 1988, ApJ, 334, 771

Walch, S., Whitworth, A. P., Bisbas, T. G., Hubber, D. A., \& Wünsch, R. 2015, MNRAS, 452, 2794

Wolfire, M. G., Hollenbach, D., \& McKee, C. F. 2010, ApJ, 716, 1191

Wolfire, M. G., McKee, C. F., Hollenbach, D., \& Tielens, A. G. G. M. 2003, ApJ, 587, 278

Wu, B., Van Loo, S., Tan, J. C., \& Bruderer, S. 2015, ApJ, 811, 56

Xie, T., Allen, M., \& Langer, W. D. 1995, ApJ, 440, 674

Zhang, Z.-Y., Henkel, C., Gao, Y., et al. 2014, A\&A, 568, A122

Zhang, Z.-Y., Papadopoulos, P. P., Ivison, R. J., et al. 2016, RSOS, 3, 160025 